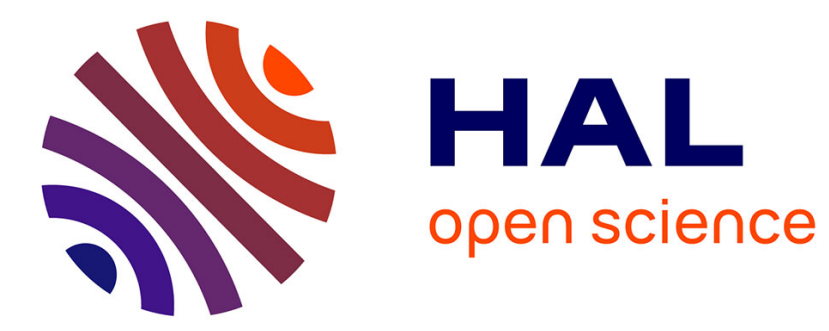

\title{
Les contrats agraires dans la Grèce antique. Bilan historiographique illustré par quatre exemples.
}

\author{
Michèle Brunet, Rougemont, Georges, Denis Rousset
}

\section{To cite this version:}

Michèle Brunet, Rougemont, Georges, Denis Rousset. Les contrats agraires dans la Grèce antique. Bilan historiographique illustré par quatre exemples.. Histoire et sociétés rurales, 1998, 9, pp.211-245. hal-01728115

\section{HAL Id: hal-01728115 \\ https://hal.science/hal-01728115}

Submitted on 9 Mar 2018

HAL is a multi-disciplinary open access archive for the deposit and dissemination of scientific research documents, whether they are published or not. The documents may come from teaching and research institutions in France or abroad, or from public or private research centers.
L'archive ouverte pluridisciplinaire HAL, est destinée au dépôt et à la diffusion de documents scientifiques de niveau recherche, publiés ou non, émanant des établissements d'enseignement et de recherche français ou étrangers, des laboratoires publics ou privés.

\section{(1) (1) $\$$}

Distributed under a Creative Commons Attribution - NonCommercial - NoDerivatives| 4.0 


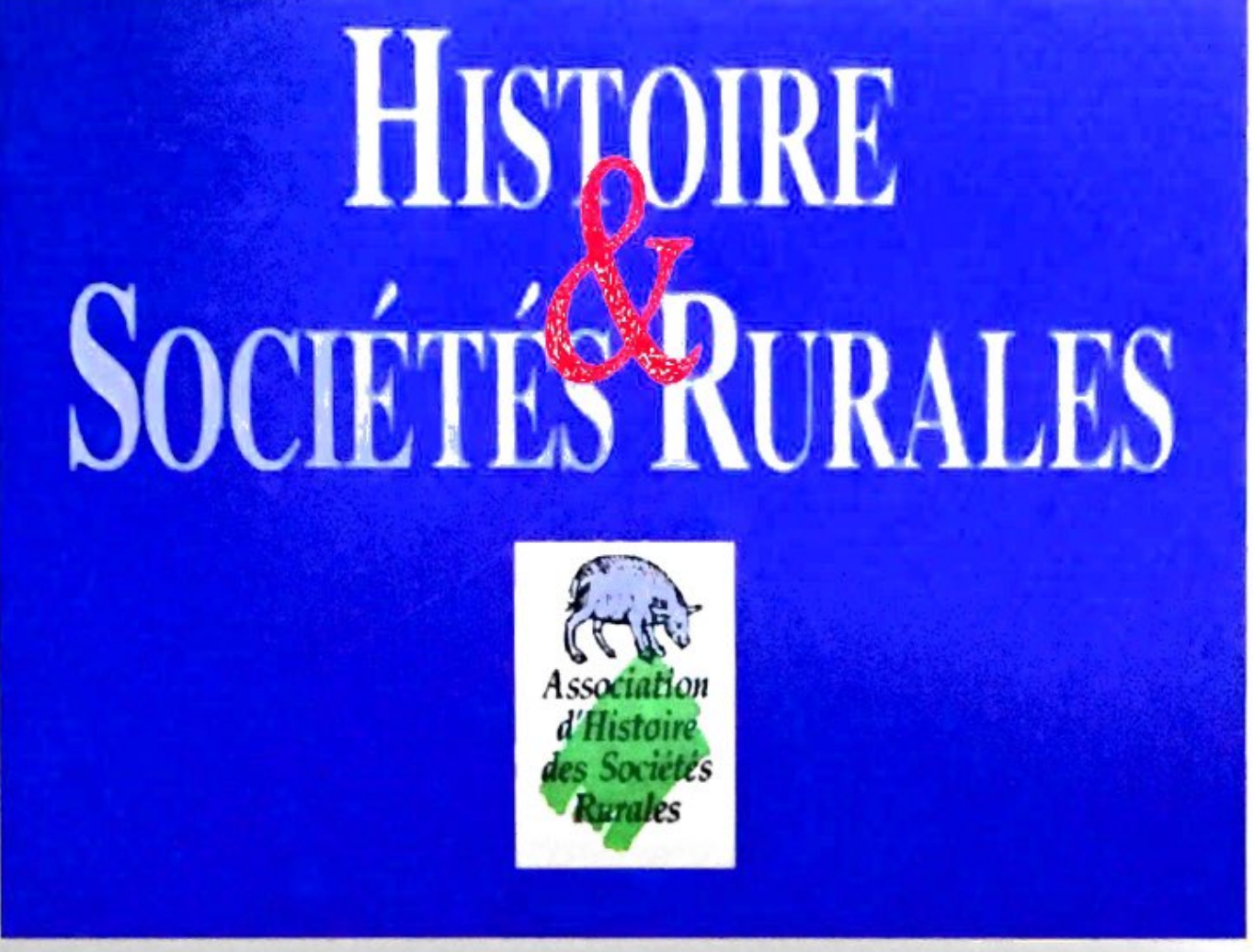

S. MAZUMDAR, Agriculture et agronomie dans la Chine Impériale

C. CHANDEZON, Paysage et économie en Asie Mineure |rr-1" siècle avant J.C. |

M. NASSIET, La diffusion du blé noir en France à l'époque moderne

B. CAMPBELL et M. OverToN, L'histoire agraire de l'Angleterre avant 1850

La Terre et les Paysans en France et en Grande-Bretagne

AUX XVII ET XVIII SIECLES.

Un parcours bibliographique critique (premiere partie)

2. Les contrats agraires dans la Grèce antique (IV-II siècle avant I.C.)

a)

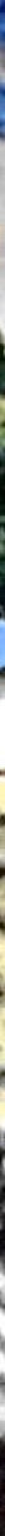




\section{Histoire ET SOCIÉTÉs RURALES}

$\mathrm{N}^{\circ} 9$ - $\mathrm{I}^{\mathrm{er}}$ semestre 1998

SOMMAIRE

Sucheta MAZUMDAR, Agriculture, agronomie et droits de petite propriété dans la Chine impériale (XVIIIe siècle avant J.-C. - I9II)

Christophe Chandezon, Paysage et économie rurale en Asie Mineure à l'époque hellénistique. A partir de quelques baux de Mylasa (IIe-Ier siècle avant J.-C.) .....

Michel NASSIET, La diffusion du blé noir en France à l'époque moderne

Bruce CAMPBELL et Mark OVERTON, L'histoire agraire de l'Angleterre avant I850. Une revue historiographique de l'état actuel de la recherche

Jean JACQUART, In Memoriam Hugues NEveUX

Jean-Marc Moriceau, La Terre et les Paysans en France et en Grande-Bretagne aux XVIre et XVIII siècles. Un parcours bibliographique critique ( ${ }^{\text {re }}$ partie) ..

\section{Sources}

Michèle Brunet, Georges RougemonT, et Denis Rousset, Les contrats agraires dans la Grèce antique. Bilan historiographique illustré par quatre exemples ....

52. Contrat-type de location des terres de Zeus Téménitès par la cité d'Arkésinè (Cyclade) (rve siècle avant J.-C.)

53. Bail pour un domaine situé à Aixoné en Attique (345 avant J.-C.)

54. Contrats de location concernant quelques domaines appartenant à Apollon, Délos (Cyclades) (177 avant J.-C.)

55. Borne-témoin d'une hypothèque prise sur un domaine, Naxos (Cyclades) (IVe siècle avant J.-C).

\section{Comptes rendus}

\section{- Ouvrages}

Marcel MAZOYER et Laurence RoUdART, Histoire des agricultures du monde. Du Néolithique à la crise contemporaine (Éric Mollard)

Yves MenEz, Une ferme de l'Armorique gauloise. Le Boisanne à Ploü̈r-sur-Rance (Côtesd'Armor) (Pierre Jaillette)

Milieux naturels, espaces sociaux : Études offertes à Robert Delort (François DuceppeLamarre)

Flore et jardins. Usages, savoirs et représentations du monde végétal au Moyen Âge (Hélène Servant)

\section{Rédacteurs en chef}

Jean-Marc MORICEAU Ghislain BRUNEL

Directeur de publication Secrétaire de rédaction

Maison de la Recherche en Sciences Humaines Archives Nationales

Université de Caen, r4032 Caen Cedex 60, rue des Francs-Bourgeois, 7514I Parrs Cedex 03 
Pouvoirs et société en Pays Albigeois, textes rassemblés par Philippe Nelrdoff et Olivier DEVAUX (Christophe Blanquie)

Philippe Le MoIng-KerRand, Pontcallec, la passionnante histoire d'un grand domaine breton (Philippe Jannoux)

Maria FuSARO, Uva passa. Una guerra commerciale tra Venezia e l'Inghilterra (I540-1640) (Mathieu Arnoux)

Christian DeSPLAT, Village de France au XVIII siècle. Autoportrait (Annie Antoine) ............

Gérard BÉAur et Philippe Minard, (dir.), Atlas de la Révolution française, volume io, Économie (Mircille Touzery)

Colette MerLIN, Ceux des villages... La société rurale dans la "Petite Montagne» jurassienne à la veille de la Révolution (Antoine Follain)

Colette Merlin, Le Pain de mêlee. Campagnes jurassiennes dans l'Ancienne France (Antoine Follain)

Anne STAMM, Retour en Millevaches. La famille, le travail, la tradition chez les paysans limousins. Récit d'une ethnologue (Antoine Follain)

Gilbert NOËL, France, Allemagne et "Europe verte" (Jean-Paul Amat)

Roger CALMES et Jean-Marc MoriceaU, (éd.), "Paysages et Structures agraires" (textes réunis et présentés par Annie Antoine), Enquêtes rurales. T. 3 (Nadine Vivier)

Yves Kinossian, (dir.), Des bois dont on fait les Vosges. Une histoire de la forêt vosgienne (Jean-Pierre Husson)

\section{- Instruments de travail}

Ulysse Louns, Pierre PAJOT, et Jean POUËSSEL, Livre de rentes de l'Hôtel-Dieu de Vernon (Christophe Maneuvrier)

Roberto Togni, Gaetano ForNI, et Francesca PISANI, Guida ai musei etnografici italiani (Corinne Beutler)

Les archives du Directoire exécutif. Inventaire des articles AF III I à sI $J$ (Dominique Godineau)

\section{- Soutenances de thèses}

Jean-Pierre VALLAT, «La Campanie rurale à l'époque romaine (Ive siècle avant J.-C./ IVe siècle après J.-C.) " (Jacques Verger)

Vincent ClÉment, «Pays et paysages de Vieille Castille (Xe-XXe siècle), étude de biogéographie historique» (Jean-Pierre Husson)

Nadine VIVIER, "Les biens communaux en France de I750 à 19I4. État, notables et paysans face à la modernisation de l'agriculture " (Jean-Michel Boehler)

Résumés

Abstracts 



\title{
SOURCES
}

\section{LES CONTRATS AGRAIRES DANS LA GRÈCE ANTIQUe Bilan historiographique illustré par quatre exemples}

\author{
Michèle BRUneT*, Georges Rougremont**, Denis ROUSSET ${ }^{* * *}$
}

$\mathrm{L}$

ARGEMENT MÉCONNUS en dehors du cercle étroit des spécialistes, les contrats agraires de l'Antiquité grecque n'ont pas retenu l'attention des historiens de la période autant qu'ils le méritent. Depuis la fin du XIXe siècle, la plupart ont été édités sans traduction ni commentaire dans les recueils d'inscriptions et, malgré quelques études consacrées aux séries régionales les plus remarquables, ces baux n’ont jamais été réunis en un corpus thématique général qui pourrait être le point de départ d'une étude historique. C'est pourquoi il nous a semblé utile d'esquisser ici un bilan, en insistant sur les caractéristiques principales de la documentation et sur l'exploitation qui peut en être faite, avant de commenter quelques exemples, sélectionnés comme témoins de la variété et de la richesse de cette source fondamentale pour l'histoire rurale de l'Antiquité grecque, un domaine de la recherche aujourd'hui en plein essor.

Dissipons d'emblée une équivoque : aucun des quatre textes que nous présentons n'est inédit; cependant, à l'occasion de cette étude, nous avons été amenés à modifier sensiblement l'interprétation traditionnelle de certains passages, et nous espérons contribuer ainsi à renouveler le commentaire de ces documents.

\section{LE CORPUS DOCUMENTAIRE}

Dans le monde grec antique, les contrats agraires étaient chose banale. Les terres données en location étaient la propriété soit de particuliers soit de collectivités, grandes ou petites : cités-États (poleis), subdivisions diverses de la polis (tantôt l'équivalent de nos collectivités locales, tantôt des groupes sociaux de nature variée), associations. Un cas en apparence particulier est celui des terres dites "sacrées ", c'est-à-dire appartenant aux dieux. Les auteurs modernes parlent souvent à leur propos de terres «appartenant aux sanctuaires». Cependant cette

\footnotetext{
* Université de Paris I, Institut d'Art et d'Archéologie, 3, rue Michelet, 750o6 PARIS.

** Université Louis Lumière-Lyon II, Institut Fernand Courby, Maison de l'Orient Méditerranéen, 7, rue Raulin, 69007 LYON.

*** Ecole Normale Supérieure, 45, rue d'Ulm, 75005 PARIS.
}

Nous tenons à remercier Marie-Claire AmOURETTI et Françoise Ruzé, qui ont apporté à notre article de judicieuses suggestions et corrections. 
expression est impropre, car un sanctuaire grec n'avait pas d'autonomie juridique par rapport à la collectivité de ses usagers. Pour les Grecs, le propriétaire d'un terrain "sacré" était donc toujours une divinité précise et les biens qu'elle possédait étaient gérés par la communauté des usagers du sanctuaire qui lui était dédié (la cité, l'une de ses subdivisions, une association, une ou des familles, dans certains cas une association ad hoc réunissant plusieurs États, comme à Delphes). Par conséquent, en pratique, les biens des dieux ne sont qu'un cas particulier des biens appartenant aux collectivités.

Il ne fait aucun doute que l'écrasante majorité des baux agricoles étaient conclus de particulier à particulier. Pourtant, à l'exception de l'Égypte grécoromaine, aucun de ces contrats privés ne nous est parvenu.

\section{Une documentation essentiellement épigraphique}

Partout dans le monde grec, les particuliers avaient l'habitude de consigner les contrats qu'ils passaient par écrit, sur un support périssable, en général du papyrus. Le papyrus, on le sait, ne se conserve que dans des conditions hygrométriques et climatiques très spécifiques. Dans l'espace habité à un moment ou à un autre de l'Antiquité par des populations de langue grecque, ces conditions ne sont guère réunies qu'en Égypte et, de fait, on y a retrouvé des contrats agricoles liant des personnes privées, rédigés sur papyrus.

Dans le reste du monde grec, les seuls baux ruraux qui soient parvenus jusqu'à nous sont donc ceux qui furent gravés sur pierre ou sur bronze (les exemples sur bronze sont évidemment peu nombreux). Il s'agit donc uniquement de contrats que les parties prenantes ont souhaité exposer en public de façon définitive, ou en tout cas très prolongée, afin de leur assurer une publicité à long terme, parallèlement à leur conservation sur un support plus éphémère dans des archives publiques ou privées. Ainsi s'explique le fait que, dans les documents épigraphiques conservés, le bailleur soit presque toujours une collectivité, soucieuse de rendre compte à ses membres des actes de sa gestion.

\section{Une documentation juridiquement hétérogène}

Le corpus des baux agricoles grecs conservés ne regroupe que quelques dizaines d'exemplaires. Ce n'est pas négligeable, mais c'est peu par rapport à d'autres séries de documents, décrets honorifiques, ex-voto, épitaphes, qui se comptent par milliers. Bien entendu, ils ne nous sont pas tous parvenus complets : depuis l'Antiquité et, parfois, dès l'Antiquité, beaucoup de pierres inscrites ont été endommagées et mutilées. En outre, du point de vue juridique, ces documents ne sont pas tous exactement des contrats de location (misthôseis) : les Grecs ignoraient les classifications juridiques rigoureuses issues du droit romain et les historiens modernes, à juste titre, n'hésitent pas à grouper pour les étudier ensemble des documents proches par leur objet, mais divers par leur forme juridique. 
Par exemple, un des deux plus anciens documents du corpus, un décret du peuple athénien rendu en 4I8-4I7 avant notre ère, ordonne de clôturer les terres appartenant à deux divinités et de les mettre en location "conformément aux dispositions en vigueur ", dispositions que nous ignorons; à la suite figurent plusieurs clauses précises, comparables à celles que l'on rencontre dans les baux ordinaires : il est donc légitime de ne pas dissocier ce texte de la série des contrats agricoles. C'est en vertu de ce principe que nous avons choisi comme quatrième exemple un document qui, bien que ressortissant stricto sensu à la série des bornes placées sur un terrain hypothéqué, fournit un témoignage sur la location de propriétés de mineurs orphelins sous le contrôle de la cité.

Les deux catégories de documents les plus fréquentes n'en sont pas moins, d'une part des contrats de location de terrains agricoles entre une collectivité (le bailleur) et un ou plusieurs particuliers (les preneurs) ${ }^{1}$, d'autre part ce qu'on pourrait appeler des contrats-types (syngraphai), où sont énumérées les conditions auxquelles une collectivité entend donner à bail des terres qui lui appartiennent ou dont la gestion lui est confiée 2 . Il arrivait qu'une version fortement abrégée de cette convention imposée d'avance à tout futur locataire fût gravée sur une borne plantée sur la propriété. Du point de vue du contenu, ces deux catégories de documents sont évidemment très voisines. D'ailleurs, le texte gravé d'un contrat-type est souvent suivi de l'indication nominative du ou des preneurs qui furent sans doute les premiers à louer la terre aux conditions fixées. Inversement, lorsque la collectivité faisait graver le texte d'un contrat passé avec un particulier, c'est vraisemblablement, dans bien des cas, parce que les clauses de ce contrat précis étaient aussi destinées à servir de modèle pour des contrats ultérieurs.

Il existe enfin une dernière catégorie de textes que l'on rattache traditionnellement à la série des baux. Il s'agit de certains passages des comptes que les administrateurs chargés par une collectivité de gérer ses biens ou ceux des dieux étaient tenus de rendre annuellement. Ces bilans d'exercice financier ont quelquefois été gravés et partiellement conservés : le cas le plus notoire est celui des comptes des administrateurs du sanctuaire d'Apollon à Délos 3 .

\section{Répartition géographique et chronologique des inscriptions conservées}

La plupart de ces inscriptions datent des quatre derniers siècles avant notre ère. Ce n'est pas surprenant : ces quatre siècles sont ceux où les inscriptions grecques sont sinon le plus nombreuses, du moins le plus variées; or les contrats agraires, nous l'avons vu, sont une catégorie d'inscriptions relativement peu représentée. Les documents plus anciens (Ve siècle) ou plus récents (sous l'Empire romain) sont rares et quelque peu atypiques.

\footnotetext{
1. Cf. source 53 .

2. Cf. source 52 .

3. Cf. source 54 .
} 
La répartition géographique des textes est très irrégulière. Ce n'est pas étonnant, et cela ne signifie pas que la pratique des baux ruraux était inconnue des régions du monde grec qui n'ont pas fourni de textes. Surtout, rappelons à nouveau que sauf exception, le document gravé est toujours, à la lettre, un document choisi parmi la masse des documents conservés en archives. Les raisons de ce choix sont toujours locales, puisque le vieux monde grec (la Grèce, les îles de la mer Égée et l'Ouest de l'Asie Mineure), qui a fourni la plupart des documents dont nous parlons, fut une mosaïque de petites communautés indépendantes les unes des autres : dans chaque cité, les lois, les traditions, les habitudes différaient. C'est sans doute la raison pour laquelle quelques cités ou quelques régions ont fourni en abondance ces documents, rares ou absents ailleurs (on observe le même phénomène pour d'autres catégories de documents, par exemple les actes d'affranchissement d'esclaves) : dans ces cités, à l'époque considérée, on avait l'habitude, ignorée ou moins enracinée ailleurs, de graver systématiquement ce type de textes.

\section{Quelques séries régionales}

C'est ainsi qu'une cité modeste de la Grèce centrale, Thespies, en Béotie, a livré une série de contrats datés entre 240 et le début du II siècle avant J.-C. La Carie, une région située au sud-ouest de la Turquie actuelle, anciennement et profondément hellénisée, a produit une série de textes datant de la fin de l'époque hellénistique (IIe-_er siècles avant notre ère), numériquement importante et qui présente des traits originaux 4 . À Délos, au centre des Cyclades, les comptes rendus des administrateurs du sanctuaire et des propriétés d'Apollon s'échelonnent entre le milieu du ve siècle et le milieu du IIe siècle avant J.-C. Athènes, enfin, a livré une très belle série de contrats et de contrats-types, assez souvent bien conservés, où le bailleur est fréquemment une collectivité locale, ou une association, ou une subdivision du corps civique - mais ce trait se retrouve dans d'autres cités (carte I).

\section{Beaucoup de documents isolés}

Cela dit, on gravait, à l'occasion, des baux un peu partout. Depuis longtemps, plusieurs îles de la mer Égée (Chios, Thasos, Kéos, Amorgos...) ont fourni chacune un ou plusieurs documents importants; plusieurs textes proviennent de régions d'Asie Mineure autres que la Carie, ou du Péloponnèse (où le sol d'Olympie a conservé, sur une petite tablette de bronze, l'un des deux documents les plus anciens de la série, très laconique), ou d'autres régions de Grèce. En Italie du Sud et en Sicile, les inscriptions et, a fortiori, les baux ruraux sont rares, sans doute parce qu'on gravait plus volontiers dans ces régions le bronze que la pierre, comme l'atteste l'un des plus beaux exemples du corpus. Ce

4. Voir l'article de Christophe Chandezon, supra, p. 35-58. 
très long texte intact sur bronze, traditionnellement appelé «Tables d'Héraclée ${ }^{5}$, fut découvert au XVIII siècle en Lucanie : vers l'an 300 avant notre ère, la cité d'Héraclée récupère des terrains sacrés qui avaient été usurpés par des personnes privées; elle fait délimiter et partager ces terres en plusieurs lots, qu'elle loue ensuite à des particuliers à des conditions que le texte énumère en détail.

Carte I.

Lieux de provenance des contrats analysés

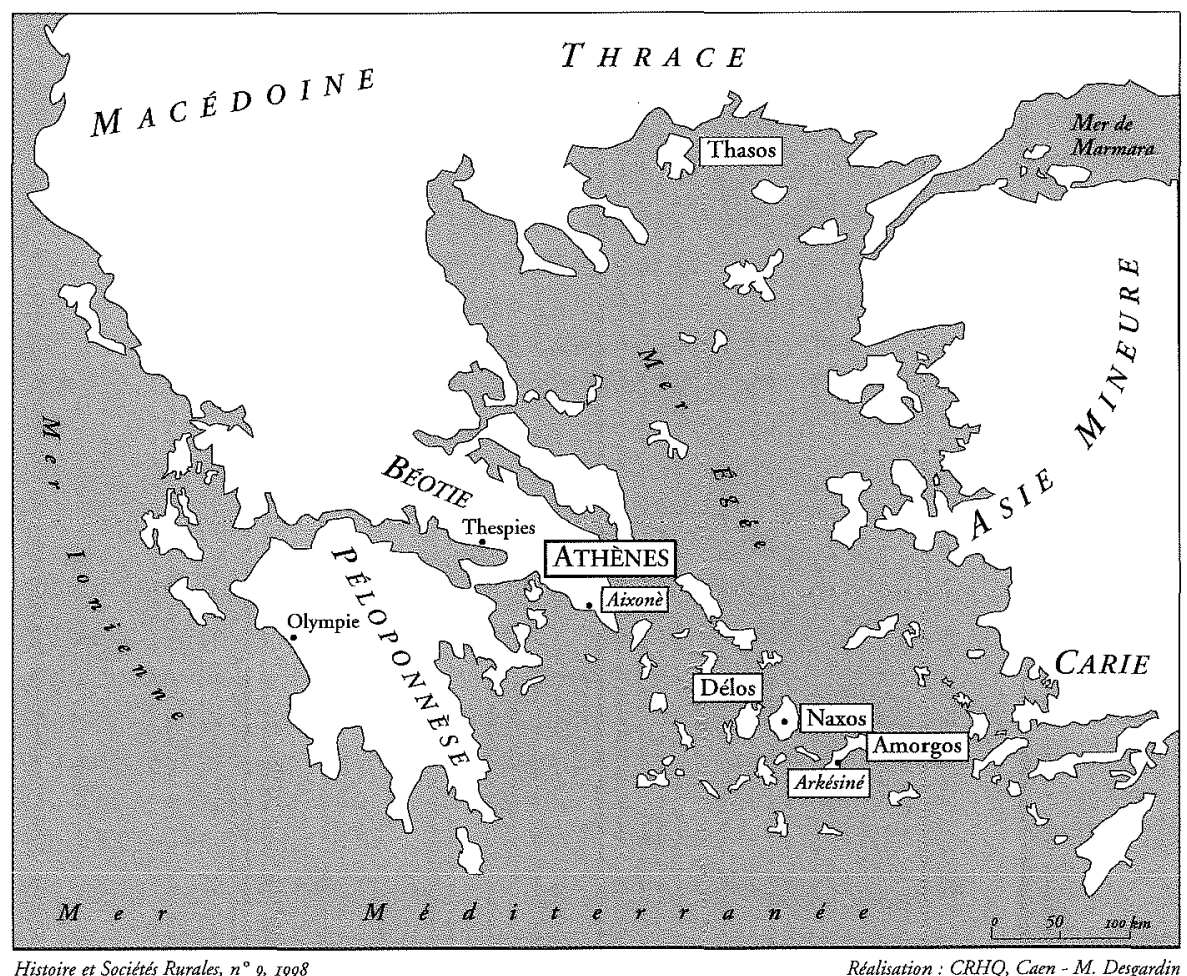

\section{TYPOLOGIE DU CONTENU DES CONTRATS}

Au-delà des différences que l'on doit attribuer aux particularismes régionaux, il existe bien une typologie commune à l'ensemble des contrats grecs antiques. Y figurent généralement, outre la date et l'identité des parties prenantes et des garants du locataire, la durée du contrat, le montant du loyer et les modalités de son versement. Les causes de sanction et d'éviction ainsi que les obligations du

5. DARESTE et alii, I894, I, p. 193-234. 
preneur (constructions s'il y a lieu, entretien du fonds et obligations culturales) sont définies de façon moins systématique.

La succession des clauses n'obéit pas toujours à une logique rigoureuse et nombre d'omissions sur des points qui pourraient sembler essentiels - comme par exemple l'emplacement et la superficie des terres - s'expliquent aisément par le contexte : ces contrats sont passés à l'intérieur de très petites communautés où tout le monde se connaît et connaît le parcellaire local ; la mention d'un lieu-dit suffit donc le plus souvent pour permettre l'identification d'une pièce de terre.

\section{Durée des contrats}

Pour la durée des baux, la décennie apparaît comme une norme, bien attestée à Athènes comme à Délos. Certes, l'on rencontre des locations de plus courte durée (cinq ans'), ainsi que des baux s'étendant sur plus d'une décennie : dans ce dernier cas, il est vraisemblable que la longueur du contrat se justifiait par les conditions particulières de mise en valeur et de rentabilisation du travail investi Mais ces contrats supérieurs à dix années demeurent des exceptions. A cette location de faible ou de moyenne durée, on oppose les baux perpétuels, souvent dits emphytéotiques ${ }^{8}$. Par le terme d'emphytéose, consacré sous l'influence du droit romain du Bas-Empire, mais que les Grecs ne paraissent pas avoir eux-mêmes employé, on désigne la concession pour toujours d'une terre, moyennant une rente très faible, concession qui laisse cependant subsister en principe les rapports de propriétaire à locataire ${ }^{9}$. De tels baux, dépourvus de terme temporel ou fixés "pour toujours", sont bien attestés dans le monde grec ${ }^{10}$. Certains d'entre eux prévoient, parmi les obligations du preneur, celle d'apporter des améliorations au fonds, en construisant ou en plantant (emphyteuein); de là le nom qui leur est souvent donnéll. Ces baux perpétuels, souvent transmissibles aux héritiers, conféraient au locataire un «droit de quasi-propriété » (Louis Gernet).

\section{Fermages}

Sil existe bien quelques documents qui mentionnent des versements en nature (c'est le cas du plus ancien bail conservé, celui d'Olympie ${ }^{12}$, daté du

6. Location des terrains sacrés d'Athéna à Héraclée de Lucanie au Ive siècle avant J.-C., DARESTE et alii, 1894, I, p. 213.

7. Cf. le commentaire du bail d'Aixonè, texte 53 .

8. Voir DARESTE et alii, I894, I, p. 229-230 et 272-274; GERNET, I980, p. 1313-I314.

9. Cf. GERNET, I980.

10. Ainsi, par exemple, à Héraclée de Lucanie, DARESTE et alii, I894, I, p. 201 et 230, et à Mylasa en Carie, cf. l'article de Christophe CHANDEZON.

11. Ainsi à Héraclée de Lucanie. Louis Gernet, 1980, a justement souligné que "les obligations particulières qui peuvent résulter de l'emphytéose entendue au sens étymologique, à savoir celles de planter $[\ldots]$, sont des obligations qui peuvent être, à l'occasion, imposées à un locataire ordinairen, sur un terme plus court.

12. DARESTE et alit, r894, I, p. 256-257. 
Ve siècle, ou des baux emphytéotiques concernant les terres de Dionysos à Héraclée de Lucanie), il paraît néanmoins invraisemblable d'interpréter cette redevance comme une part de fruit qui serait versée dans le cadre d'un métayage, impliquant la fourniture par le bailleur de l'outillage et des semences : aucun texte grec ne témoigne en effet de l'existence de telles pratiques. Les baux grecs sont donc des contrats de fermage, dans lesquels le montant d'un loyer fixe est quasiment toujours exprimé en numéraire. Les versements sont parfois répartis en plusieurs termes dans l'année, qui correspondent généralement aux temps forts de l'activité agricole méditerranéenne (moisson, vendange, récolte des olives); mais très souvent, c'est en juillet-août, donc juste après les moissons, que la totalité du fermage était exigée.

Le mode le plus usuel d'attribution des contrats était l'adjudication. Toutefois, dans la majorité des cas, les montants indiqués ne peuvent guère être exploités, en l'absence de données contemporaines suffisantes. Et même lorsqu'on dispose d'une série de fermages répartis sur une assez longue période, comme à Délos, il s'avère bien difficile d'interpréter la courbe de leurs fluctuations, dont les causes peuvent avoir été tout aussi bien locales que conjoncturelles.

\section{Contraintes culturales et obligations du preneur}

Nombre de contrats n'imposent aucune contrainte quant au mode de jouissance ou d'exploitation du fonds loué. Hormis les cas où le contrat concerne la bonification d'une terre récemment acquise ou à l'abandon, et comprend par conséquent un cahier des charges détaillé, la plupart des documents ne comportent que quelques prescriptions, tout à fait banales si l'on considère leur fréquence, concernant l'entretien des terres et des éventuels bâtiments. Compte tenu de la rotation rapide des locataires, l'objectif recherché par les bailleurs n'était donc pas l'amélioration du fonds, destinée à accroître sa productivité, mais la simple préservation de sa valeur. Souvent sont uniquement explicitées les obligations du preneur pour la dernière année du bail (notamment la mise en jachère de la moitié des terres conformément à l'assolement biennal, de règle dans le monde grec), afin d'assurer une transmission équitable au locataire suivant et, partant, de maintenir la valeur locative du bien. Un passage de Théophraste 13 illustre cette pratique courante : «Les Thasiens, lorsquils donnent leurs terres agricoles en location, ne se soucient pas des autres années et souhaitent même une mauvaise exploitation. Mais pour la dernière année (du bail), ils rédigent des clauses en vue de la reprise ». La sous-exploitation apparaissait donc préférable à une mise en culture forçant les sols et les plantations.

13. De causis plantarum, II, II, 3, commenté par François SALVIAT, I972, p. 372-373. 


\section{L'INTERPRÉTATION DES CONTRATS AGRAIRES}

L'interprétation de ces documents se heurte aux limites qu'imposent les caractéristiques du corpus que nous venons d'exposer : l'isolement de la majorité des textes, la prédominance quasi exclusive des contrats émanant de corps publics constituent les limites les plus évidentes, auxquelles s'ajoute, en dépit de quelques heureuses exceptions, l'impossibilité de replacer les baux dans leur contexte économique, faute de données précises concernant les superficies mises en location, le prix des terres, les prix de vente des produits agricoles et leur évolution respective dans chacune des cités concernées par un document ${ }^{14}$.

C'est donc uniquement pour les séries les plus fournies, en particulier celles d'Athènes et de Délos, que le commentaire peut être poussé, grâce à la confontation avec d'autres sources - épigraphiques, littéraires ou matérielles - qui éclairent plus largement le contexte social, économique et environnemental.

\section{Place et rôle de l'affermage des terres agricoles}

Les objectifs recherchés par les collectivités-bailleurs sont assez clairs : la rente foncière servait principalement à couvrir leurs dépenses de fonctionnement, achat des bêtes de sacrifice, réparations des bâtiments ou organisation de fêtes et banquets. Toutefois, lorsqu'elle atteignait un niveau élevé comme à Délos, elle pouvait être employée à d'autres fins comme, par exemple, le prêt.

\section{Une pratique réservée à un groupe social restreint}

Dans les cas où les preneurs sont connus par d'autres sources, il s'avère que ce sont des citoyens aisés, possédant le plus souvent par ailleurs des terres qu'ils exploitent en faire-valoir direct ${ }^{15}$. L'affermage de terres par les collectivités intervenait donc presque toujours à l'intérieur d'un groupe socialement restreint, qui disposait à la fois d'un capital foncier et de moyens de production, c'est-à-dire d'une main-d'œuvre servile. On constate qu'en Attique les contrats étaient souvent conclus à une échelle très locale, entre proches et voisins d'un même dème. Dans le cadre de cités où les terroirs étaient généralement de dimensions réduites et exploités à des fins essentiellement vivrières, avec quelquefois, localement, des secteurs de spécialisation développés pour le commerce d'exportation, les terres offertes à la location par des collectivités formaient une réserve de terrains agricoles dont l'importance pouvait être non négligeable : ainsi on a estimé qu'en

14. Pour paraphraser Moses FINLEY citant A. H. M. JONES, faut-il rappeler cette «ignominieuse vérité ", qui risque de jeter le discrédit sur l'histoire de l'Antiquité : "Il n'y a pas de statistiques anciennes" (FINLEY, 1982, p. 697).

15. Rappelons que dans la Grèce antique, le droit de propriété foncière était en principe réservé aux citoyens et que la terre était la principale source de richesse. Qu'il s'agisse de faire-valoir direct ou indirect, les domaines importants étaient généralement confiés à un régisseur (épitropos) de condition servile. 
Attique, leur superficie représentait environ le dizième des terres cultivables ${ }^{16}$. L'accès à ces terres ne bénéficiait cependant qu'aux citoyens déjà bien nantis, qui usaient de la location comme d'un moyen d'augmenter temporairement leurs revenus par la vente de surplus agricoles. Les historiens s'accordent aujourd'hui pour reconnaittre que les conduites économiques des Grecs de l'Antiquité différaient fondamentalement des nôtres : bien que le réinvestissement productif ne soit pas à exclure, les gains recherchés par ce moyen étaient sans doute souvent destinés à financer des dépenses ponctuelles parfois lourdes, au profit de la collectivité, comme les liturgies ${ }^{17}$, ou personnelles, comme le versement d'une dot lors du mariage des filles. De ce fait, la location de terres agricoles peut être considérée comme un moyen de compléter, voire d'éviter l'emprunt garanti par l'hypothèque foncière, qui constituait le mode de financement privilégié pour ce type de dépenses 18 .

Ainsi, d'après les principales études ${ }^{19}$ consacrées aux baux consentis par des collectivités, il apparaît que la mise en location de cette catégorie de biens-fonds n'était destinée ni à favoriser l'accès à la terre des citoyens non propriétaires ou des étrangers domiciliés dans la cité (métèques), ni à permettre à de petits propriétaires d'atteindre un meilleur niveau de production pour leur autoconsommation ${ }^{20}$. Cette pratique apparaît donc comme essentiellement conservatrice, renforçant les solidarités entre nantis tout en conférant au preneur un surcroît de prestige social.

Par contraste, les quelques allusions à des contrats entre particuliers que l'on rencontre dans les plaidoyers attiques du IVe siècle montrent que l'affermage des biens-fonds privés concernait d'autres catégories de la population, et pouvait offrir un accès à la terre à des citoyens pauvres, voire à des affranchis et à des métèques 21 .

\section{Paysages agraires et systèmes de culture}

Il serait illusoire d'espérer reconstituer à partir des contrats agraires le détail des paysages ruraux, des parcellaires, voire la configuration exacte des bâtiments de ferme ${ }^{22}$. Néanmoins, les prescriptions qui figurent dans certains baux sont,

\section{ANDREYEV, I974, p. 43.}

17. Dépenses que les plus riches citoyens devaient assumer à tour de rôle dans de nombreuses poleis; les plus connues sont la chorégie (entretien d'un chœur dans le cadre des concours théâtraux) et la triérarchie (armement et équipage d'un navire de guerre), mais beaucoup d'autres concernaient des fêtes religieuses.

18. FINLEY, I95I.

19. ANDREYEV, 1974, p. 25-46, et OSBORNE, 1988, p. 289-292.

20. On considère que les propriétés avaient en moyenne entre 4 et 5 ha de superficie, ce qui permettait à une famille de cinq personnes de couvrir ses besoins annuels en pratiquant une judicieuse polyculture.

21. OSBORNE, 1988, p. 304-319.

22. Cf. sur cette question l'article nuancé de Christophe CHANDEzon, ici-même, et les remarques de BRUNET, 1990. 
en dehors des écrits des agronomes grecs, les seules sources qui nous donnent des renseignements concrets sur les systèmes de culture et les techniques agricoles habituelles dans les cités. La polyculture méditerranéenne typique associant céréales, oliviers et vignoble est à peu près partout de règle, même si le mode de rédaction des contrats aboutit généralement à laisser dans l'ombre les emblavures - en dehors du respect de la jachère biennale, elles ne nécessitent aucun soin particulier -, et à mettre l'accent sur les plantations, en obligeant au remplacement des arbres et des ceps de vigne morts. Les contrats de bonification ou de remise en culture de fonds à l'abandon n'ont sans doute pas été l'occasion d'introduire à proprement parler des «innovations» en matière agricole; on constate cependant une concordance frappante entre certaines clauses des baux et les conseils des agronomes du Ive siècle, tel Théophraste, qui témoignent d'un souci d'adapter au mieux les cultures aux diverses qualités des sols ${ }^{23}$. Les fouilles de champs viennent aujourd'hui confirmer la réalité de ces pratiques.

En outre, à un autre niveau, il apparaît que dans les cités méditerranéennes où les contraintes de l'écosystème sont fort nombreuses (fréquence des reliefs de pente, violence des précipitations saisonnières), l'affermage des terres contribuait à sa manière à une saine gestion du finage au bénéfice de communautés civiques qui avaient comme idéal l'autarcie vivrière. La dispersion des parcelles dans l'ensemble des terroirs, leurs dimensions souvent réduites créaient une solidarité de fait entre les exploitants soumis aux mêmes contraintes. Il était impératif de préserver le fragile équilibre des pentes par l'entretien régulier des murs de terrasses et des voies de circulation des eaux pluviales, a fortiori lorsqu'il existait des réseaux d'irrigation par canaux comme ceux que l'on vient de découvrir à Délos 24 ; de ce fait, toute terre à l'abandon devenait rapidement source de nuisances pour les parcelles voisines 25 et risquait de compromettre le fonctionnement de l'ensemble du versant. Sans doute cet aspect des choses n'est-il pas à négliger pour expliquer l'intervention des autorités officielles dans l'affermage des terres d'orphelins : outre la sauvegarde du patrimoine des mineurs, c'était aussi la protection des terroirs qui était implicitement en jeu. Cependant, il ne semble pas que les contrats de location aient été utilisés par les cités grecques comme moyens d'une bonification systématique de terres marginales ou conquises, signe d'une conception et d'une pratique de l'État fort différente de celles des Romains.

23. Cf. par exemple SALVIAT, 1993, commentant des baux rhodiens d'environ 200 avant J.-C., qui prévoient la plantation d'un vignoble de cru en plaine et sur coteaux, avec des vignes sur arbres (figuiers) et des cultures intercalaires en bandes, bel exemple de coltura promiscua planifiée.

24. Brunet et Poupet, 1998.

25. Cf. Demosthène, Contre Calliclès, LV, ir. Le plaidoyer fut prononcé à l'occasion d'un conflit entre propriétaires voisins, provoqué par la construction d'un mur de clôture barrant le lit d'un torrent saisonnier; la négligence d'un ancien exploitant est invoquée pour expliquer les dommages causés par les eaux divagantes. 


\section{BIBLIOGRAPHIE}

\section{Études générales}

Dareste, Rodolfe, Haussouldier, Bernard, et Reinach, Théodore, Recueil des inscriptions juridiques grecques, I, Paris, 1894, p. 193-274. Recense les baux ruraux grecs connus à cette époque, et reproduit, traduit en français et commente avec clarté les textes les plus importants.

Gurraud, Paul, La propriété foncière en Grèce jusquià la conquête romaine, Paris, Imprimerie nationale, I893, p. 42I-445.

OSBORNe, Robin, "Social and Economic Implications of the Leasing of Land and Property in Classical and Hellenistic Greece ", Chiron, I8, 1988, p. 279-323.

\section{Baux d'Athènes}

AndreyeV, V. N., "Some Aspects of Agrarian Conditions in Attica in the Fifth to Third Centuries B. C. ", Eirene, I2, I974, p. 5-46.

BeHrend, Dietrich, Attische Pachturkunden, Munich, 1970.

Walbank, Michael B., The Athenian Agora, XIX, Inscriptions, Princeton, 1991, p. 147-207.

\section{Baux de Thespies}

Osborne, Robin, "The Land Leases from Hellenistic Thespiai : a re-examination", in Argoud, Gilbert, et Roesch, Paul, (éd.), La Béotie antique, Paris, I985, p. 317-323.

\section{Baux de Carie}

Voir dans le présent numéro, supra, p. 54-56, la bibliographie rassemblée par Christophe Chandezon.

\section{Autres références}

BRUNET, Michèle, "Contribution à l'histoire rurale de Délos aux époques classique et hellénistique ", Bulletin de correspondance hellénique, II4, 1990, p. 669-682.

BRUnet, Michèle, et Poupet, Pierre, "Le paysage agraire de Délos dans l'Antiquité», Journal des Savants, 1998 (à paraître).

FINLEY, Moses I., Studies in Land and Credit in Ancient Athens, 500-200 BC. The Horos-Inscriptions, New Brunswick, New Jersey, Rutgers, I951;

—, "Le document et l'histoire économique de l'Antiquité», Annales ESC, XXXvile année, s/6, septembre-décembre 1982, p. 697-713.

GERNET, Louis, "Aspects du droit de propriété en Grèce», Annali della Scuola normale superiore di Pisa, 10, 1980, p. 1309-1328.

SALVIAT, François, "Bail thasien pour un terrain planté", Bulletin de correspondance hellénique, 96, 1972, p. 363-373;

—, "Le vin de Rhodes et les plantations du dème d'Amos", in AmoureTtT, Marie-Claire, et Brun, Jean-Pierre, (éd.), La production du vin et de l'huile en Méditerranée, Athènes, École française d'Athènes, Bulletin de correspondance hellénique, Supplément, XXVI, I993, p. I5I-I6I. 


\section{2. \\ IVe siècle avant J.-C. \\ Contrat-type de location des terres de Zeus Téménitès par la cité d'Arkésinè à Amorgos (Cyclades)}

Principales éditions : Homolle, Théophile, Bulletin de correspondance hellénique, 16, 1892, p. 276 294; DelamarRe, Jules, Revue de Philologie, 190I, p. 165-188; Inscriptiones graecae, XII, 7, 62; DIT-

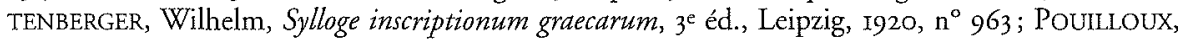
Jean, Choix d'inscriptions grecques, Paris, Belles Lettres, 1960, $\mathrm{n}^{\circ} 35$.

N.B. : Conformément aux conventions de l'épigraphie grecque, les parties de texte illisibles ou manquantes sont signalées et éventuellement restituées entre [] et les lettres qui ne sont que partiellement déchiffrables sont "pointées", par ex. yọy. Pour faciliter le repérage des articulations du document, nous avons introduit une numérotation en caractères gras $(\mathrm{I}, 2$, etc.) dans le texte et dans la traduction. Il en va de même pour les trois textes suivants.

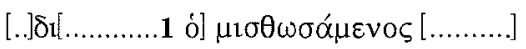

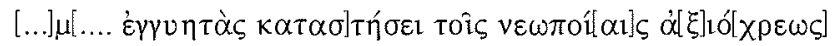

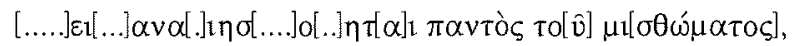

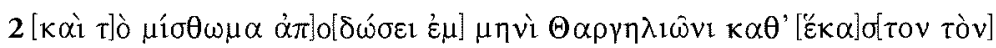

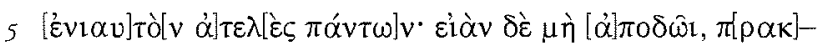

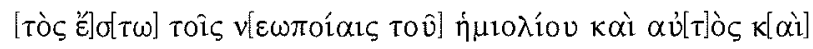

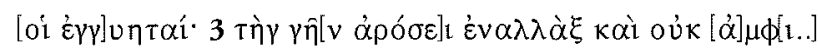

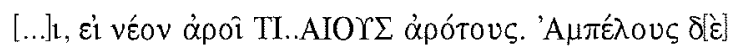

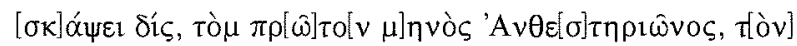

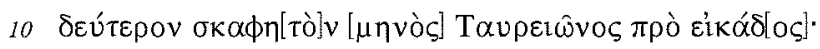

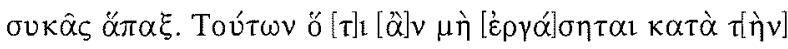

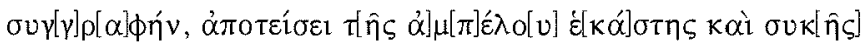

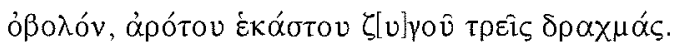

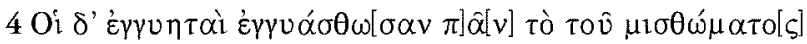

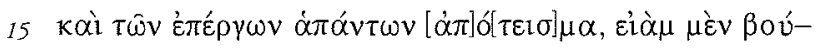

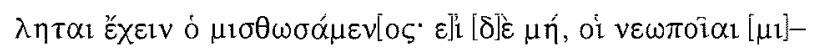

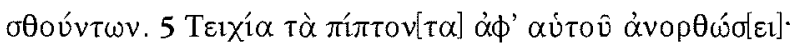

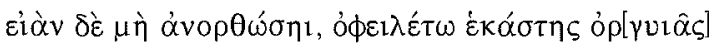

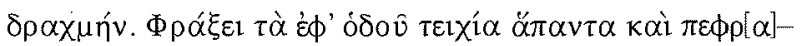

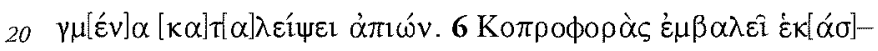

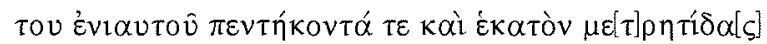

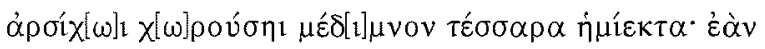

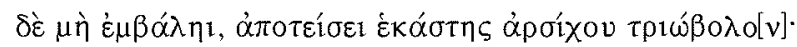

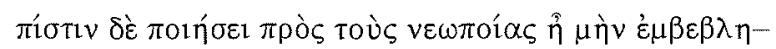

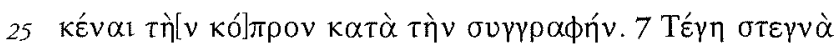

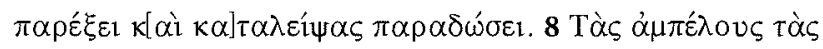

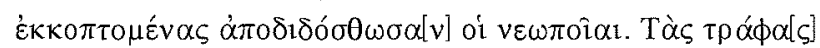

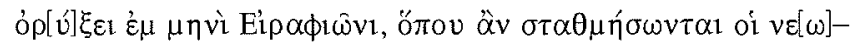




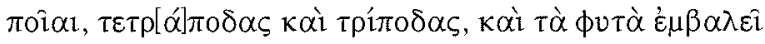

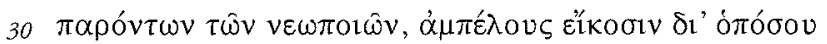

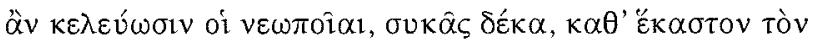

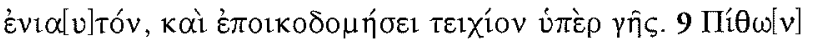

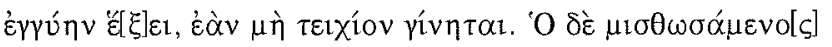

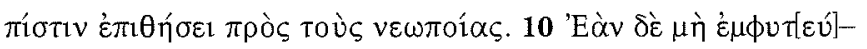

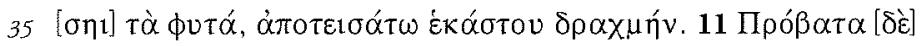

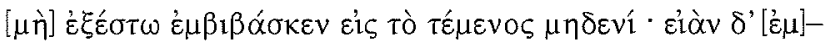

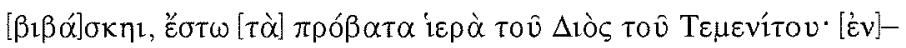

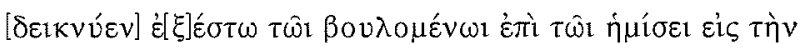

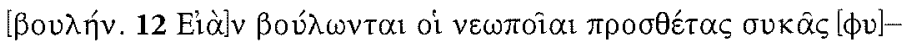

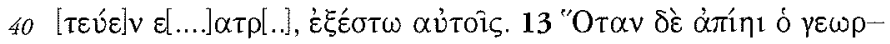

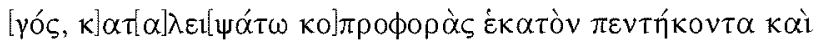

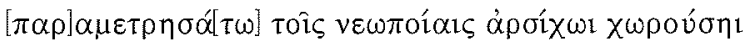

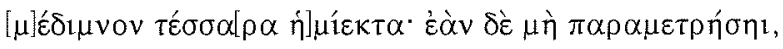

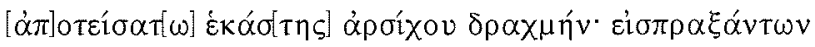

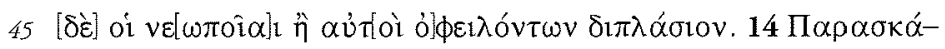

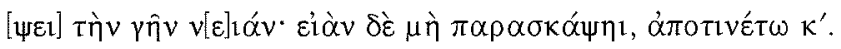

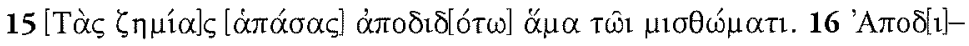

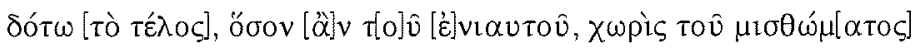

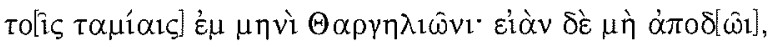

so $\pi \rho \alpha \kappa \tau$ tò

(restes de six lignes)

\section{Traduction}

1. [...] Le preneur [...] fournira aux néopes ${ }^{1}$ des cautions sûres [...] [...] la totalité du loyer;

2. et il paiera le loyer au mois de Thargéliôn ${ }^{2}$, chaque année, indépendamment de tout impôt ${ }^{3}$. S'il y manque, lui-même ou ses cautions seront passibles d'une majoration égale à la moitié du montant du loyer 4 , à recouvrer par les néopes.

3. Il labourera la terre par assolement biennal et il ne [..?]5 les champs ensemencés. Les

1. Les néopes sont les magistrats de la cité-État d'Arkésinè chargés de la gestion des sanctuaires.

2. Les noms et l'ordre de succession des mois, dans la Grèce antique, variaient d'une cité à l'autre. Le calendrier en usage dans la cité d'Arkésinè est incomplètement connu, mais il semble proche du calendrier attique, dans lequel Thargéliôn correspond à mai-juin.

3. Sur le paiement de l'impôt, voir ci-dessous le $\$$ I 6 .

4. Littéralement : ils paieront une fois et demie le montant du loyer.

5. La pierre est très endommagée à cet endroit. On avait conjecturé qu'il pourrait y être ques-

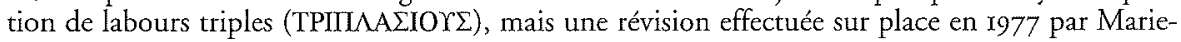


vignes, il les piochera deux fois par an, la première au mois d'Anthestériôn ${ }^{6}$, la seconde au mois de Taureiôn, avant le vingtième jour du mois; les figuiers, une seule fois. S'il n'effectue pas un de ces travaux de la façon prévue par le contrat-type, il paiera, pour chaque pied de vigne et pour chaque figuier, une amende d'une obole ${ }^{7}$, et, pour chaque zugon $^{8}$ de terre ensemencée, une amende de trois drachmes?.

4. Les cautions garantiront en totalité le paiement du loyer et celui des amendes pour non-exécution de travaux, si le preneur veut continuer à jouir du bail; s'il y renonce, les néopes remettront le bail en adjudication.

5. Le preneur relèvera à ses frais les murs qui s'effondrent. S'il ne les relève pas, il devra une drachme par orgyie ${ }^{10}$. Il devra garnir de protections tous les murs du côté du chemin et les laisser garnis en partant ${ }^{11}$.

6. Il épandra chaque année 150 métrètes 12 de fumier en se servant d'un panier contenant un médimne et quatre hémiectes ${ }^{13}$. S'il n'épand pas ce fumier, il paiera une amende de trois oboles par panier manquant. Il attestera par un serment prêté devant les néopes qu'il a bien procédé à l'épandage conformément au contrat-type.

7. Il assurera l'étanchéité des couvertures et il les badigeonnera avant de les rendrel4.

8. Les pieds de vignes coupés seront mis en vente par les néopes. Le preneur creusera les tranchées au mois d'Eiraphiôn, aux endroits mesurés par les néopes - dimension des tranchées : 4 pieds sur 3 -, et il y placera les plants en présence des néopes, à savoir 20 pieds de vigne, plantés aux intervalles fixés par les néopes, et to figuiers, cela chaque année, et il construira un mur au-dessus du sol.

9. [?], s'il n'y a pas de mur; le preneur s'y engagera par serment devant les néopes.

10. S'il n'effectue pas les plantations, il paiera une amende d'une drachme par pied non planté.

11. Défense est faite à quiconque d'introduire du bétail sur le domaine. En cas d'infraction, les bêtes seront confisquées au profit de Zeus Téménitès. Qui le voudra pourra dénoncer au Conseil 15 les infractions éventuelles; le dénonciateur touchera la moitié de l'amende infligée ${ }^{16}$.

Françoise Boussac et Georges Rougemont a permis d'éliminer la possibilité d'une telle restitution, sans pour autant parvenir à une nouvelle lecture fournissant un sens cohérent.

6. Vraisemblablement février-mars. On ignore par contre à quels mois correspondent Taureiôn (ligne suivante) et Eiraphiôn, $\$ 8$.

7. L'obole est une unité monétaire de faible valeur. Dans l'ignorance où l'on est des prix et des salaires dans cette cité et à cette date, tout calcul d'équivalence serait illusoire.

8. Mesure de superficie, de valeur inconnue.

9. $\mathrm{I}$ drachme $=6$ oboles.

10. Mesure de longueur, approximativement $1,75 \mathrm{~m}$. Il doit s'agir de la longueur de mur non réparée.

11. C'est-à-dire à la fin du bail.

12. À Athènes, le métrète vaut un peu moins de 40 litres.

13. Soit, à Athènes, près de 80 litres. Environ 75 de ces paniers seraient donc nécessaires à l'opération.

14. C'est-à-dire juste avant la fin du bail.

15. De la cité.

16. Pratique banale dans les cités grecques. 
12. Si les néopes veulent planter des figuiers supplémentaires [...], ils le pourront.

13. Quand le cultivateur s'en ira ${ }^{17}$, il laissera 150 mesures de fumier; il remettra ce fumier aux néopes en le mesurant au moyen d'un panier contenant un médimne et quatre hémiectes. S'il manque à cette obligation, il paiera une amende d'une drachme par panier manquant. Les néopes sont chargés du recouvrement de l'amende; s'ils y manquent, ils paieront eux-mêmes le double de l'amende.

14. Le preneur défoncera la jachère à la pioche. S'il y manque, il paiera une amende de 20 drachmes.

15. Le preneur paiera l'ensemble des amendes en même temps que le loyer.

16. Il paiera le montant annuel de l'impôt, indépendamment du loyer, aux trésoriers ${ }^{18}$, au mois de Thargéliôn. S'il y manque, il sera passible d'une majoration égale à la moitié du montant de l'impôt, à recouvrer par les trésoriers.

M. B. et G. R.

\section{Commentaire}

L'aspect actuel du terroir : environnement et vestiges antiques

Le lieu de trouvaille de la pierre est assez exactement connu et il permet - chose raredes hypothèses plausibles sur la localisation et l'aspect du terroir concerné. En effet, avant d'être utilisé pendant de nombreuses années comme pierre de seuil pour une église, le bloc de marbre inscrit avait été exhumé dans un champ à quelques minutes de marche au nord d'un village qu'on appelait tout simplement, il y a cent ans, to chorio ("le village»), et qui a été rebaptisé au XXe siècle Arkésini. Malgré ce nom «savant », le village en question n'occupe pas le site de la ville antique d'Arkésinè : celle-ci est parfaitement identifiée et se trouve à une heure et demie de marche de là. En revanche il est certain que ce village et, partant, le lieu de trouvaille de l'inscription, se trouvaient sur le territoire de la cité-État d'Arkésinè : le domaine et, vraisemblablement, le sanctuaire de Zeus Téménitès se trouvaient donc dans la campagne, à bonne distance du centre urbain. En effet, à cause des dimensions de la pierre (I,50 $\mathrm{m}$ de hauteur, près de $50 \mathrm{~cm}$ de largeur, I5 $\mathrm{cm}$ d'épaisseur) et de son poids, il est peu probable qu'elle ait été déplacée sur une longue distance entre l'Antiquité et sa redécouverte au xrxe siècle : selon un usage bien attesté, elle était probablement érigée dans l'enceinte même du sanctuaire.

À Amorgos comme dans tout l'archipel des Cyclades, les terres cultivables sont particulièrement rares. Or le village moderne domine précisément une des rares petites plaines de l'île : un minuscule bassin à fond plat, en pente très légère vers le Nord-Ouest, suspendu à un peu moins de $200 \mathrm{~m}$ d'altitude et sans contact direct avec la mer. De plus, approximativement dans le secteur où l'inscription fut découverte, au bord de cette petite plaine, se trouve la ruine antique la mieux conservée de l'île : un ouvrage fortifié construit en pierres de taille, composé d'une tour carrée de $9 \mathrm{~m}$ de côté, flanquée d'un enclos de $27 \mathrm{~m} \times 15 \mathrm{~m}$ délimité par de hauts murs percés, comme ceux de la tour, de meurtrières.

17. A la fin du bail.

18. De la cité. Les néopes perçoivent le loyer $(\$ 2)$, les trésoriers perçoivent l'impôt. 
Des ouvrages de ce genre sont fréquents dans les campagnes des cités grecques antiques. Leurs fonctions ont fait l'objet de longues discussions (fermes fortifiées? tours de guet?), compliquées par le fait qu'ils ne sont pas tous aussi bien conservés que celuici. Parmi les fonctions possibles de l'édifice dont nous parlons, la plus évidente est défensive : sans être capable de soutenir un siège en règle ou d'abriter longtemps une population même peu importante, il pouvait toutefois offrir une protection suffisante contre une attaque de courte durée menée avec des moyens «légers». Or les sources écrites de cette époque attestent en très grand nombre à la fois l'insécurité ordinaire des campagnes (guerres, brigandage, piraterie - cette dernière particulièrement banale dans l'Archipel -) et la présence de petites forteresses destinées à protéger contre elle les cantons un peu trop éloignés des centres urbains qui, de leur côté, étaient tous fortifiés; la ville d'Arkésinè ne fait pas exception à la règle.

Il est donc loisible et plausible de supposer que le domaine de Zeus Téménitès se trouvait quelque part dans cette petite plaine isolée et sur les pentes qui l'environnent, près d'un hameau placé sous la protection d'un fortin où les habitants pouvaient se réfugier pour quelques heures en cas d'attaque imprévue. (G. R.)

Depuis sa découverte, ce document a fait l'objet de plusieurs éditions, commentées ou non, qui ne sont cependant pas parvenues à fournir un sens satisfaisant pour quelques passages. Certaines difficultés de compréhension du texte $(1.7-8 ; 1.32-33)$ sont liées à des problèmes de déchiffrement de la pierre; seule une révision dans de bonnes conditions matérielles pourrait éventuellement permettre des améliorations de lecture et, en son absence, nous nous abstiendrons de tout nouveau commentaire sur ces lignes. En revanche, à la lumière des recherches récentes sur le paysage grec antique et grâce aux développements que connaît l'histoire des techniques agricoles, il est aujourd'hui possible de proposer une nouvelle traduction de certaines clauses mal comprises. Nous nous attacherons donc essentiellement à justifier ces nouvelles interprétations, qui permettent de montrer que la succession des clauses du contrat n'est pas hasardeuse, mais obéit à un plan logique.

\section{La rédaction du contrat}

Les premières lignes du contrat (syngraphè, 1. I2 et 25), qui contenaient vraisemblablement la date et la durée du bail, sont perdues et nous avons laissé de côté les dernières lignes, trop mal établies. À la suite d'une première clause précisant la date de versement du fermage, en un terme annuel au mois de juin (donc juste après la moisson qui s'effectue en mai dans les Cyclades), figure une première série de clauses ( $\$ 3$ à 7 ) relatives à la conduite et à l'entretien courant de la propriété ; puis sont détaillées ( $\$ 8$ à I2) les opérations de renouvellement des plantations qui devront être effectuées par tranches annuelles. Enfin, avant de revenir sur les modalités financières ( $\$$ Is et I6), le contrat précise les obligations du preneur lors de la dernière année du bail. Chacune des clauses prévoit, en cas d'infraction, une amende proportionnelle.

La confrontation avec d'autres documents du même type et avec les écrits des agronomes révèle la précision du vocabulaire employé par les rédacteurs de ce texte; pour la désignation des composantes du bien-fonds comme pour celle des travaux prescrits, ils usent des termes techniques adéquats. 


\section{Composition de la propriété}

Cultures. Selon le principe de l'alternance biennale, la terre arable (gê) est divisée en deux soles, l'une ensemencée (arotoi, 1. 8 et I3), l'autre en jachère (néia, 1. 46); les plantations arbustives (ta phyta, 1. 29) se répartissent en vignes et en figuiers. Mais, tout en se conformant à la pratique de la polyculture comme toutes les exploitations grecques de l'Antiquité, ce domaine "sacré» (téménos, 1.36) présente la double particularité de ne pas associer une petite activité d'élevage à la culture des terres et de ne posséder aucun olivier. Ce dernier trait, partagé avec les propriétés sacrées déliennes, est à mettre au compte d'un particularisme régional : l'olivier résiste mal aux vents du Nord violents (les meltems) qui soufflent sur l'archipel cycladien durant plusieurs mois de l'année; sa culture semble avoir été peu développée dans la région durant l'Antiquité et, aujourd'hui encore, elle reste limitée aux versants protégés du vent.

Constructions. En matière d'entretien des constructions, l'unique exigence porte sur la préservation de l'étanchéité des couvertures, $\$ 7$. Le verbe employé (kataleiphô, 1. 26) permet de comprendre que sont concernés des toits en pente couverts de tuiles, qui étaient imperméabilisés chaque année à la saison sèche par l'application d'un badigeon de poix ${ }^{19}$. A cause de l'absence de toute autre précision concernant les bâtiments ${ }^{20}$, on peut supposer que le bien-fonds ne comportait aucune maison vouée à la résidence permanente, et que ces constructions étaient de simples remises abritant l'outillage.

Les teichia. On traduit généralement ce diminutif (formé sur le mot teichos qui nomme ordinairement le mur d'enceinte d'une ville) par "petit mur, muret». Mur de clôture ou mur de soutènement d'un champ en terrasse? Comme le prouve l'observation des campagnes cycladiennes contemporaines, ces fonctions sont toutes deux envisageables a priori, et un seul et même mur peut les avoir remplies simultanément. De fait, depuis la mise en évidence récente de l'existence des terrasses de culture dans la Grèce antique ${ }^{21}$, les commentateurs s'interrogent sur leur dénomination et, parmi d'autres, le terme teichion pourrait convenir pour désigner leurs soutènements appareillés 22 . L'ambiguïté apparaît bien à la $1.17, \$ 5$ : on enjoint au locataire de remonter les «murets qui s'effondrent", et dans ce cas, il peut tout aussi bien être question des dentelles de pierre sèche clôturant les parcelles que des soutènements de champs en gradins. Toutefois, l'opération plus spécifique qui est demandée à la l. Ig ne semble pouvoir concerner que des murs de clôture ${ }^{23}$. Le verbe employé, qui apparaît fréquemment dans un contexte militaire, indique que les murets doivent être «couverts, garnis de défenses» : selon une pratique encore très courante dans les campagnes grecques de nos jours, et d'une efficacité redoutable, il s'agit très probablement de placer sur la crête des murs des buissons d'épineux, afin d'empêcher toute intrusion des bêtes et des passants dans la propriété. Cette interprétation permet ainsi de mieux comprendre pourquoi on exige de laisser les

19. Sur cette opération très banale pour l'entretien des pièces de bois et des couvertures de tuiles dans le sanctuaire d'Apollon à Délos, cf. HellmanN, 1992, p. 37-42.

20. Notamment à propos des portes, cf. ci-après le commentaire du document 54 .

21. BRUNET, I990; RACKHAM et MOODY, 1992.

22. D'autant plus que certains contrats agraires français du XVIe et du XVII siècle emploient communément le mot de "muraille" pour parler des murs de terrasse : cf. BLANCHEMANCHE, 1990, exemples p. 269-270.

23. Cf. FOXHALL, 1996, p. 49-50.

Histoire et Sociétés Rurales, $\mathrm{n}^{\circ}$ 9, $\mathrm{I}^{\mathrm{er}}$ semestre $\mathrm{I} 998$, p. 2II-245. 
murs "garnis» lors de la reprise du fonds : le preneur pourrait souhaiter récupérer ces matériaux de protection pour les utiliser ailleurs ou s'épargner un travail désagréable la dernière année.

Il est plus malaisé de saisir la fonction exacte du muret dont la construction est exigée à la l. 32 : s'agit-il d'un muret bas sans fondations («sur le sol»), qu'il faudrait élever en relation avec l'opération de plantation des vignes et des figuiers pour la protection des jeunes plants? Quoi qu'il en soit, on peut supposer que les moellons utilisés pour cette construction proviennent de l'épierrage des tranchées creusées pour les plantations.

\section{La conduite du domaine}

Emblavures. Malgré les difficultés de déchiffrement que soulèvent les lignes 7 et 8 ; il faut sans doute admettre à la suite de la plupart des commentateurs qu'il est question du respect de la mise en culture "en alternance" (enallax), donc de l'assolement biennal : un tel système de culture allant pratiquement de soi24, cette prescription s'explique sans doute par l'interdiction qui l'accompagnait, et qui malheureusement nous échappe. En revanche, il nous semble que l'opération concernant la jachère exposée aux lignes 45-46 n'a généralement pas été bien comprise. On pensait qu'il était demandé au preneur de "délimiter la jachère par un fossé »25, sans pour autant être en mesure d'expliquer l'utilité d'une telle entreprise. Or l'emploi du verbe paraskaptô indique en réalité un tout autre travail, par ailleurs bien connu : il s'agit de procéder au labour de défoncement de la jachère, qui consiste à ouvrir à la pioche ou à la bêche (skaptố) des tranchées parallèles ${ }^{26}$ dans le champ et à les combler en déversant les déblais de l'une dans l'autre. Comme le précise Xénophon dans son petit traité l'Économique27, il est préférable d'effectuer ce travail à la main plutôt qu'à l'araire, utilisé pour les autres labours (labour d'été et labour d'automne couplé aux semailles ${ }^{28}$ ), car l'instrument manuel permet de retourner la terre plus profondément. Notre traduction se fonde en outre sur le parallélisme avec les travaux de piochage à effectuer au pied des vignes et des figuiers ${ }^{29}$, exprimés par le verbe skaptô (l. 9 et Io), tandis qu'un autre verbe (orussô) est employé pour désigner le travail de creusement des tranchées de plantation (I. 28) : à n'en pas douter, cette nuance de vocabulaire recouvre à la fois une différence de gestes, d'outillage et donc de finalité des travaux. De surcrôt, notre interprétation explique pourquoi le manquement à cette obligation lors de la dernière année du bail est taxé d'une lourde amende (20 drachmes) : le labour de défoncement devait impérativement être effectué

24. Voir cependant AMOURETTI, 1986, p. 51-57, qui évoque la possibilité d'assolements triennaux et les utilisations de la jachère pour des cultures dérobées, qui pourraient précisément être interdites ici.

25. Voir par exemple la traduction de PoullLoux, I960, p. I38.

26. Ce qui expliquerait peut-être le préfixe para accolé au verbe skaptô. Ce verbe composé n'est pas attesté en dehors de cette inscription.

27. XVI, I5.

28. Sur cette question de la préparation des sols, cf. AMOURETTI, 1986, p. 57-58.

29. Il est demandé d'appliquer au sol du vignoble deux façons culturales, l'une en février-mars (Anthestériôn), l'autre en Tauréiôn. Si l'on se réfere à la culture traditionnelle du vignoble cycladique telle qu'elle se pratique encore à Santorin, ce second piochage destiné à aérer le sol quand les rameaux ont atteint 15 à $20 \mathrm{~cm}$ doit intervenir en avril-mai : cf. Le Santorin de Santorin, Athènes, Fondation Fany Boutari, 1995, p. I2I-I25. 
au printemps, donc quelques mois avant le départ du preneur, qui pouvait de ce fait être tenté de se dispenser d'un travail pénible dont il ne serait pas le bénéficiaire; mais en l'absence de ce labour préparatoire, la rotation correcte des soles serait perturbée et, par conséquent, la valeur locative du domaine s'en trouverait diminuée.

Furnure. Les prescriptions portant sur l'épandage du fumier ne nous arrêteront pas longtemps ${ }^{30}$. Théophraste consacre de longs développements à l'usage raisonnable qu'il conseillait d'en faire tant pour les plantations que pour les emblavures, et l'on constate de fait que c'est sur les quantités à répandre dans l'année que portent les directives : elles correspondent environ à $6 \mathrm{~m}^{3}$ au total, ce qui est peu. Néanmoins, l'obligation de laisser en partant une provision annuelle (\$13) appelle un commentaire ; elle s'explique en effet par le caractère particulier de ce domaine où, contrairement à la plupart des exploitations antiques, aucune activité d'élevage n'était pratiquée en association avec le système de culture. Moutons et chèvres (probata, 1. 35) sont ici expressément bannis de la totalité du domaine, alors que d'ordinaire les contrats agraires ${ }^{31}$ interdisent seulement l'accès du petit bétail aux vignobles et aux vergers, ménageant ainsi la possibilité d'une vaine pâture sur la jachère et sur les chaumes. Par conséquent, aucun fumier n'étant produit sur la propriété de Zeus Téménitès, le preneur devait en acquérir à l'extérieur et le transporter à ses frais. On imagine bien que le locataire sur le départ devait être tenté de s'économiser ces dépenses, totalement ou partiellement (en fraudant sur les quantités), d'où le soin avec lequel les autorités contrôlaient à la fois la présence et le volume du stock au moment de la reprise.

\section{Les modes de plantation du vignoble et des figuiers}

Le preneur doit procéder au remplacement du vignoble par tranches annuelles $(\$ 8$, coupe des anciennes vignes, plantation de jeunes ceps). La plantation concomitante de vignes et de figuiers pourrait laisser supposer qu'il s'agit ici de vignes sur arbres, un type de mariage fréquent et bien attesté tant par les sources grecques que latines ${ }^{32}$, mais une telle conduite n'est concevable que dans les endroits bien protégés des vents, et nous ignorons si tel était le cas des parcelles concernées : de nos jours, dans les Cyclades battues par les vents étésiens, ce sont des vignes rampantes que l'on rencontre le plus souvent. Si les clauses du contrat ne fournissent aucun indice susceptible d'éclairer ce point, elles permettent toutefois de se représenter parfaitement le mode de plantation préconisé. À l'évidence, la traduction du terme traphè (1. 27) a posé un problème aux précédents commentateurs et a entrâné des erreurs sur l'interprétation de l'opération requise 33 .

Certes, ce terme technique est peu usité, mais son emploi dans le bail du dème d'Amos (situé dans les possessions continentales de Rhodes), récemment republié34, lève

30. Sur ce point, voir les analyses de SALVIAT, 1972 ; AMOURETTI, 1986, p. 62-63; et pour la vigne, BILLIARD, I913 (1997), p. 328-344.

31. Comme par exemple la hiéra syngraphè délienne.

32. Cf. BlLliard, 1913 (1997), p. 366-368.

33. Bien que les tranchées de plantation aient déjà été évoquées par certains, elles ont souvent été confondues avec des «fosses» de plantation, et l'on a parfois imaginé qu'elles étaient destinées à accueillir des bornes de pierre délimitant les parcelles plantées : cf. PoullLouX, 1960, p. 137, à la suite d'HOMOLLE, I892, p. 285-286.

34. Cf. SALVIAT, 1993, p. 152, 1. 8 du contrat qui concerne également la plantation d'un vignoble 
aujourd'hui toute ambiguïté. Dans ces deux occurrences très similaires, il s'agit bien de creuser des tranchées de plantation selon une technique décrite par Théophraste 35 : à défaut de défoncer la totalité de la surface quand le sol est aride et sec, «on pratiquera des tranchées (taphroi) aussi profondes et larges que possible, et on piochera près des plants, afin qu'en été ils aient le moins de chaleur possible». Toutefois, à la différence des directives très précises du bail rhodien, qui définissent les espaces à respecter entre les tranchées et entre les plants, le présent contrat laisse aux autorités surveillant les travaux la liberté de les déterminer chaque année au coup par coup $(\$ 8)$ : un tel pragmatisme s'explique sans doute par une configuration irrégulière du terrain et de la propriété qui oblige à une disposition des plants moins rigoureuse que celle qu'autorise le domaine rhodien. Seules deux indications chiffrées figurent ici, qui concernent un point essentiel pour le développement correct des plants, la largeur et la profondeur à donner aux tranchées : 4 pieds sur $3( \pm 1,20 \mathrm{~m} \text { sur } 0,90 \mathrm{~m})^{36}$. Quels que soient les espacements entre les 20 ceps et les Io figuiers repiqués chaque année - qu'il est impossible de restituer du fait de notre ignorance du type de conduite adopté -, on conçoit que ce travail de renouvellement du vignoble, qui nécessitait de nombreuses journées de travail pénible, soit programmé sur plusieurs années.

Compte tenu de la multiplicité des exigences du bailleur, qui imposaient ces lourdes tâches dont la rentabilité n'était pas immédiate, la durée de ce bail devait très probablement être plus longue que les dix années habituelles en Grèce antique pour les contrats de location. Comme à Aixonè en Attique (cf. document 53), une quarantaine d'années apparaît comme une durée plausible, afin que le contrat demeure intéressant pour un preneur qui ne pouvait espérer tirer profit, avant que les nouveaux plants ne rendent, que des récoltes de céréales, complétées par le maigre produit d'un vignoble âgé en diminution constante.

M. B.

BIBLIOGRAPHIE

Amouretti, Marie-Claire, Le pain et l'buile dans la Grèce antique, Paris, Les Belles-Lettres, "Annales Littéraires de l'Université de Besançon, 328", 1986, 379 p.

BILLIARD, Raymond, La vigne dans l'Antiquité, Lyon, Librairie Lardanchet, I9I3, rédité avec un avant-propos d'André TCHERNIA, Laffite Reprints, 1997, 560 p.

Blanchemanche, Philippe, Bàtisseurs de paysages. Terrassement, épierrement et petite hydraulique agricole en Europe (XVIT-XIXe siècle), Paris, Éditions de la Maison des Sciences de l'Homme, I990, IX-329 p.

BRUNET, Michèle, "Terrasses de culture antiques : l'exemple de Délos, Cyclades ", Méditerranée, 7I, 1990, p. 5-II.

FOXHALL, Line, "Feeling the earth move : cultivation techniques on steep slopes in classical antiquity ", in SHIPLEY, Graham, et SALMON, John, (éd.), Human Landscapes in Classical Antiquity, Londres, Routledge, 1996, p. 44-67.

couplé à des figuiers, et où le creusement d'une tranchée est prescrit avec le même verbe, orussô, et le même substantif, trapha.

35. De causis plantarum, III, I2, traduction reprise de François SALVIAT, 1993, p. I53.

36. La comparaison avec toutes les données chiffrées disponibles montre que la largeur de la tranchée est toujours supérieure à sa profondeur. 
Hellmann, Marie-Christine, Recherches sur le vocabulaire de l'architecture grecque d'après les inscriptions de Délos, Athènes, École française d'Athènes, BEFAR, 278, 1992, 472 $\mathrm{p}$.

RackHaM, Oliver, et Moody, Jennifer, "Terraces", in Wells, Berit, (éd.), Agriculture in Ancient Greece, Stockholm, Paul Aströms Förlag, I992, p. I23-130.

SALVIAT, François, "Bail thasien pour un terrain planté», Bulletin de correspondance hellénique, 96, 1972, p. 363-373;

-, "Le vin de Rhodes et les plantations du dème d'Amos ", in AmouretTi, Marie-Claire et BRuN, Jean-Pierre, (éd.), La production du vin et de l'buile en Méditerranée, Athènes, École française d'Athènes, Bulletin de correspondance hellénique, Supplément, XXVI, 1993, p. 151-161.

\section{3. \\ 345 avant J.-C. \\ Bail pour un domaine situé à Aixonè en Attique}

Principales éditions : BOECKH, Auguste, Corpus inscriptionum graecarum, $\mathrm{I}$, Berlin, $\mathrm{I} 828, \mathrm{n}^{\circ} 93$; Dareste, Rodolfe, Haussoullier, Bernard, et ReinaCH, Théodore, Recueil des inscriptions juridiques grecques, I, Paris, I894, $\mathrm{n}^{\circ}$ XIII bis (avec une traduction dont nous nous inspirons); DITTENBERGER, Wilhelm, Sylloge inscriptionum graecarum, $3^{e}$ éd., Leipzig, 1920, $\mathrm{n}^{\circ}$ 966; KIRCHNER, Johannes, Inscriptiones Atticae Euclidis anno posteriores, II, 2, Berlin, 1931, $\mathrm{n}^{\circ} 2492$; PLEKET, Henry Willy, Epigraphica I. Texts on the Economic History of the Greek World, Leiden, I964, $\mathrm{n}^{\circ} 42$.

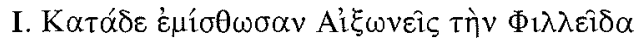

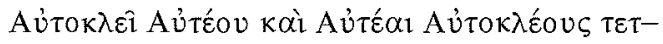

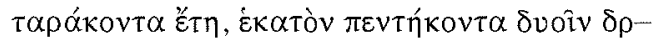

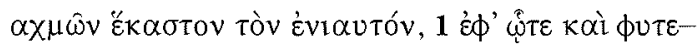

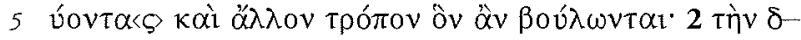

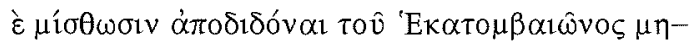

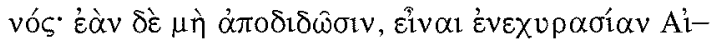

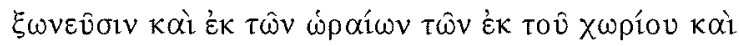

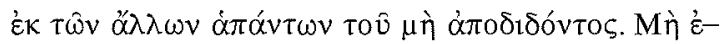

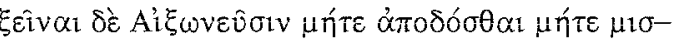

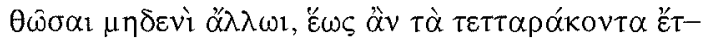

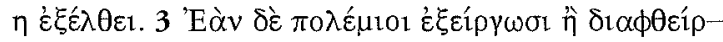

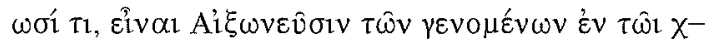

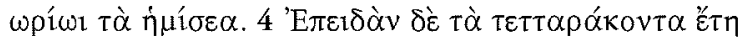

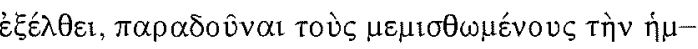

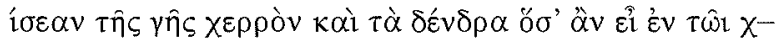

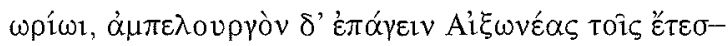

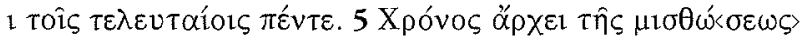

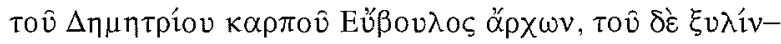

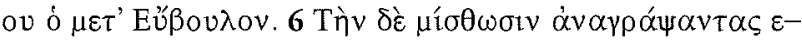




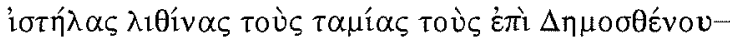

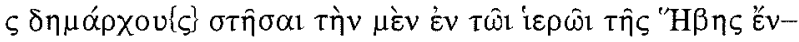

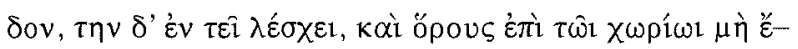

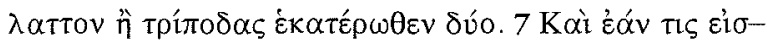

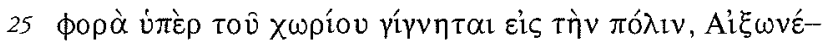

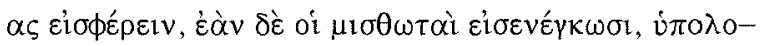

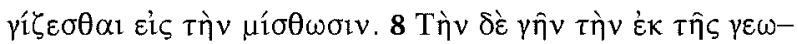

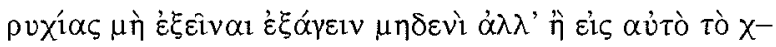

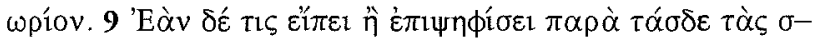

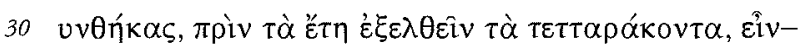

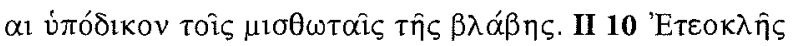

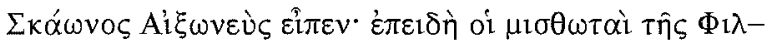

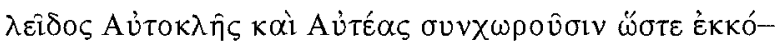

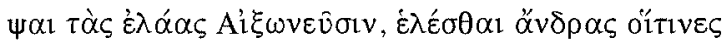

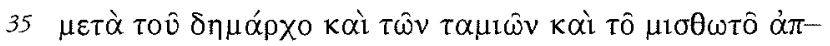

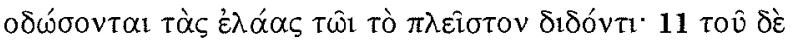

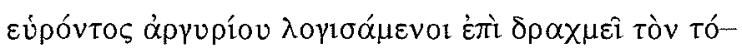

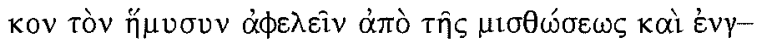

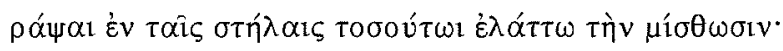

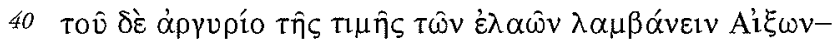

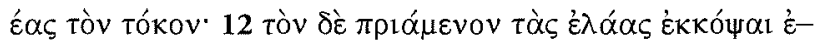

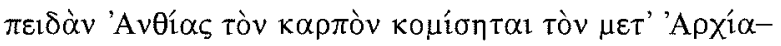

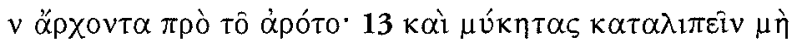

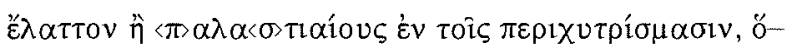

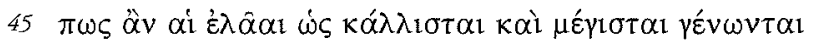

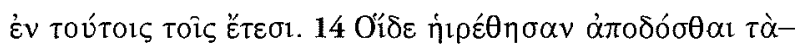

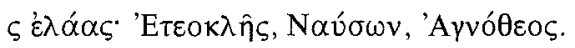

\section{Traduction}

I. Conditions auxquelles les Aixonéens ont loué la Philléis à Autoclès, fils d'Autéas, et Autéas, fils d'Autoclès, pour quarante ans, moyennant 152 drachmes par an.

1. Les preneurs la cultiveront à leur guise, en étant cependant tenus d'y faire des plantations.

2. Ils paieront le loyer au mois d'Hékatombaiôn ${ }^{2}$; s'ils ne le paient pas, les Aixonéens auront le droit de pratiquer une saisie sur la récolte du domaine et sur tous les autres biens du débiteur ${ }^{3}$. Les Aixonéens n'auront pas le droit ni de vendre ni de louer le

1. Nom du domaine.

2. Ce mois, le premier de l'année dans le calendrier athénien, correspond à juillet.

3. Alors que les locataires sont au nombre de deux, on passe ici curieusement au singulier : cette

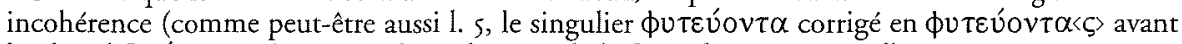
le pluriel $\beta o u ́ \lambda \omega v \tau \alpha \mathfrak{l})$ est sans doute la trace de la formule stéréotypée d'un contrat-type. 
domaine à personne d'autre jusqu'à l'expiration des quarante années.

3. S'il y a quelque trouble ou destruction par fait de guerre, les Aixonéens auront la moitié des fruits du domaine.

4. À l'expiration des quarante années, les preneurs rendront la moitié de la terre en jachère et autant d'arbres qu'en compte le domaine; les Aixonéens enverront un vigneron dans les cinq dernières années.

5. Le bail commence, pour la récolte des céréales, avec l'archontat d'Euboulosí, pour la récolte des plantations, avec l'archontat suivant.

6. Les trésoriers en charge sous le démarque 5 Démosthénès feront transcrire le contrat de location sur des stèles de pierre et feront placer l'une dans le sanctuaire d'Hébè à l'intérieur, l'autre dans la galerie; ils feront placer aussi des bornes hautes de trois pieds au moins ${ }^{6}$ sur le bord du domaine, deux sur chacun des deux côtés.

7. Si la cité frappe le fonds d'une contribution extraordinaire, les Aixonéens la verseront; si les preneurs la versent, ils la déduiront du loyer.

8. Il est interdit à quiconque d'emporter ailleurs que sur le domaine même la terre provenant des fouilles.

9. Si quelqu'un fait ou met aux voix une proposition contraire à ces clauses avant l'expiration des quarante années, il sera passible d'une action en dommage de la part des preneurs.

II. 10. Étéoclès, fils de Scaon, du dème d'Aixonè, a fait la proposition ${ }^{7}$ : attendu que les preneurs de la Philléis Autoclès et Autéas consentent à laisser couper les oliviers au profit des Aixonéens, on choisira trois citoyens qui, accompagnés du démarque, des trésoriers et du preneur ${ }^{8}$, vendront les oliviers au plus offrant;

11. la moitié des intérêts, calculés au taux d'une drachme $(=\mathrm{r} 2 \%)^{9}$, sera soustraite au loyer et l'on inscrira sur les stèles que le loyer est diminué d'autant; les Aixonéens recevront les intérêts de l'argent provenant de la vente;

12. l'acheteur ne coupera les oliviers qu'après qu'Anthias ${ }^{10}$ aura fait la récolte sous l'archontat suivant celui d'Archias'11, avant le labour;

13. il laissera des souches hautes d'au moins une palme ${ }^{12}$ dans les cuvettes, afin que les oliviers deviennent aussi beaux et grands que possible dans ces années.

4. Euboulos, archonte "éponyme» donnant son nom à l'année, exerça sa charge en $345 / 4$.

5. Magistrat local, à l'échelon du dème.

6. Soit au moins $90 \mathrm{~cm}$.

7. Ici commence le texte d'un amendement qui modifie le premier contrat.

8. Plutôt que de voir dans ce singulier une formule stéréotypée que l'on aurait oublié d'adapter au cas d'Autoclès et d'Autéas, on peut considérer que le locataire ici concerné est l'actuel occupant du domaine, Anthias, nommé $\$$ I2, 1. 42.

9. Il faut en effet comprendre "une drachme (par mois, par mine)», soit I2\% l'an. C'est le taux normal à cette époque dans une cité grecque.

10. Nom de l'actuel locataire du domaine.

11. Archias, archonte en $346 / 5$, est le prédécesseur d'Euboulos.

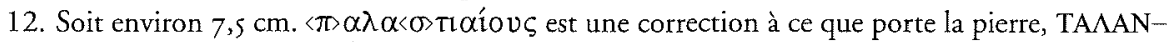
TIAIOY $\Sigma$, qui ne donne pas de sens satisfaisant. 
14. Ont été choisis comme commissaires pour la vente des oliviers : Étéoclès, Nauson, Hagnothéos.

\section{Commentaire}

\section{Institutions et droit}

À la différence du document précédent qui énumère en un contrat-type les conditions imposées à tout locataire, cette inscription présente les conditions auxquelles deux particuliers, le père et le fils, louent le domaine dit Philléis au dème d'Aixonè, c'est-àdire à la subdivision de la cité d'Athènes formée par les Aixonéens ${ }^{13}$. Le document n'est cependant pas un contrat rédigé en bonne et due forme par les deux parties, mais juxtapose une première convention définissant les conditions générales du bail (I $\$ \$$ I-9) et un décret additionnel qui la modifie (II $\$ \$$ IO-I3). Cet assemblage composite n'est pas étonnant dans les documents officiels grecs. Ajoutons que la rédaction de la convention fut finalement modifiée, avant la gravure du texte sur la pierre, pour faire apparaître non seulement le montant définitif du loyer, diminué à la suite de la coupe des oliviers, mais le nom de l'archonte, le magistrat de la cité qui permet de dater le document ${ }^{14}$ : alors que le décret additionnel ne mentionne que "l'archonte suivant Archias» (archonte en $346 / 5$ ), dont le nom n'était pas encore connu (\$ I2), la convention mentionne finalement de façon explicite le nom d'Euboulos, l'archonte de l'année 345/4 (\$5). Il est donc vraisemblable que ce bail fut négocié à la fin de l'archontat d'Archias, soit au printemps 345, peu avant l'entrée en charge au mois d'Hekatombaiôn (juillet) ${ }^{15}$ de son successeur, Euboulos.

Ce bail présente certains traits fréquents dans les textes de cette nature, particulièrement les baux athéniens : loyer calculé à l'année et payable en une fois, le premier mois de l'année, en juillet $\left(\$_{2}\right)$; au cas où la cité imposerait une contribution exceptionnelle, ne relevant donc pas de la fiscalité locale du dème, celle-ci est à la charge du dème, non des preneurs $(\$ 7)$; enfin, il est interdit d'emporter la terre arable du domaine $(\$ 8)$. Le document s'écarte en revanche de la norme par la durée inhabituelle du contrat, quarante années, alors que les autres baux connus engagent le plus souvent les parties pour dix ans; le preneur n'est par ailleurs tenu de fournir ni hypothèque ni garant.

Quant au domaine, nous en ignorons la superficie, comme souvent dans ces documents; il devait se présenter comme un triangle ou un rectangle assez allongé, puisque l'on juge bon de placer des bornes sur deux côtés seulement $(\$ 6)$; on peut penser que ces bornes, semblables aux bornes de propriété qui ont été retrouvées, avaient pour fonction de rappeler par une inscription l'identité du propriétaire, le dème, pour éviter que ne naisse à ce propos une incertitude en raison de la longueur du bail.

\section{Cultures et calendrier du bail}

S'agissant des obligations culturales imposées aux preneurs, ce bail est, sauf pour les plantations, assez clair, et peu contraignant : il laisse les preneurs libres de choisir la

13. Le démarque et les trésoriers $\$ 6$ et ro sont des magistrats locaux du dème.

14. Nous suivons sur ce point BEHREND, 1970.

15. Équivalence conventionnelle, avec des possibilités de décalage telle ou telle année. 
nature et le rythme de leurs cultures $\left(\$_{\mathrm{I}}\right)$, à l'exception de la dernière année où la moitié de la terre du domaine devra être en jachère $\left(\$_{4}\right)$ : cette pratique de l'assolement est fréquente, comme aussi pareille clause prévoyant la nécessité de préparer le terrain pour le successeur ${ }^{16}$.

Comment en revanche comprendre les indications des clauses $\$$ I et 4 relatives aux plantations? La première oblige les preneurs à "faire des plantations": s'agit-il d'accroître la part dévolue aux arbres et aux vignes? La clause $\$ 4$ stipule cependant que le domaine devra compter à l'issue du bail «autant d'arbres» : il pourra en compter plus, mais ce n'est pas obligatoire. S'il ne s'agit donc pas d'augmenter le nombre d'arbres, doit-ce être le cas des vignes? Il est justement question du vignoble dans la même clause $\$ 4$ : «le dème enverra un vigneron dans les cinq dernières années ». On imagine cependant mal qu'un ouvrier spécialisé soit envoyé à seule fin de vérifier le nombre de pieds de vigne. En réalité, le vigneron doit effectuer une opération technique d'entretien qui est jugée nécessaire, pendant les cinq dernières années, à la remise du domaine à son propriétaire et à une nouvelle location ${ }^{17}$. Rien dans notre texte $n$ 'indique donc que les preneurs doivent augmenter le nombre des vignes. Par conséquent, il nous semble que l'obligation de «faire des plantations" concerne simplement le remplacement des arbres et des vignes qui meurent.

C'est d'ailleurs le renouvellement des oliviers que règle le décret additionnel, qu'il faut analyser en détail. Au moment de la passation du bail, les oliviers du domaine devaient être vieux et pour améliorer le fonds sur le long terme, on décide de renouveler la plantation, en utilisant la technique du recépage. Les arbres sont coupés, mais on laisse leurs souches en place, hautes d'une palme $( \pm 7,5 \mathrm{~cm})^{18}$. Les rameaux qui renaîtront de ces souches irriguées dans des cuvettes (perichytrisma) ne donneront des fruits qu'environ quinze ans plus tard, et une pleine production après trois ou quatre décennies : ainsi «les oliviers deviendront aussi beaux et grands que possible dans ces (40) années» (\$13). On voit donc que la durée inhabituelle du bail, quarante années, est liée au renouvellement complet de l'olivette.

Le recépage des oliviers entraînera pour les preneurs une lourde charge d'entretien pendant maintes années, sans les récoltes correspondant à ce travail. En compensation de ce manque à gagner, ils auront droit, non pas à une partie du produit de la vente du bois des arbres, ni aux intérêts que rapportera le placement de cette somme, mais à une déduction sur leur loyer d'un montant égal à la moitié de ces intérêts (\$ II). Il reste à déterminer à quelle date on procédera à la coupe des oliviers ( $\$ \mathrm{I} 2$ ). On laissera au pré-

16. Voir ci-dessus le document d'Arkésinè, $\$$ I3 , et le commentaire afférent.

17. On ne peut se satisfaire de l'explication de DARESTE et aliz, 1894, p. 264 : "pour surveiller le fermier et l'empêcher d'emprunter à la vigne», ni de celle de BEHREND, 1970, p. 81 : "Während der letzten $s$ Jahre dürfen die Aixoneer zur Anlage einer Rebenkultur einen Winzer auf dem Grunstück haben ».

18. Sur le recépage et pour tout ce paragraphe, cf. AMOURETTI, 1986, p. 59, et 1992, ainsi que BERTRAND et BRUNET, 1993, p. 176-177. Marie-Claire AMOURETTI, 1992, p. 80 et fig. p. 8I, distingue deux techniques de recépage : "On coupe le tronc, d'où l'on dégagera ensuite les rejets [...]. On

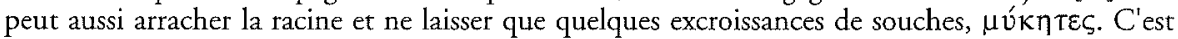
la méthode proposée dans le bail d'Aixonè». Il paraît difficile de décider si mukètes, qui paraît être un hapax dans un tel contexte (sens général : "champignon »), désigne la souche elle-même ou des excroissances de celle-ci. 
cédent locataire, Anthias, dont le bail se termine avec l'archontat d'Archias, soit le printemps 345, le temps de cueillir les fruits des oliviers qui résultent de son travail : c'est pourquoi il fera la récolte des olives sous le successeur d'Archias, à savoir Euboulos, en octobre-novembre 345. L'adjudicataire du bois des oliviers interviendra ensuite, mais avant le labour effectué par Autoclès et Autéas, de façon à ne pas gêner la culture des céréales entre les oliviers. Si le cycle est comme souvent en Grèce celui des céréales d'automne, il est donc vraisemblable que la récolte des olives sera immédiatement suivie de la coupe des arbres, elle-même suivie du labour précédant les semailles (sens précis d'arotos), qui ont lieu en novembre ${ }^{19}$.

C'est ce calendrier qui peut nous aider à expliquer la clause du $\$ s$ : les nouveaux locataires ont droit dès la première année du bail, celle d'Euboulos, aux céréales, récoltées au printemps 344 (et semées quelques mois plus tôt); mais c'est seulement l'année suivante (344/343) qu'ils auront la jouissance des plantations, en en recueillant la récolte (xylinos karpos). Comme il est exclu en raison de la coupe des oliviers qu'il y ait des olives, l'expression xylinos karpos désigne la vigne et la production des arbres fruitiers : ce sont donc en définitive ces fruits qui sont réservés jusqu'au printemps 344 au locataire précédent, Anthias. Au terme du bail, Autoclès (s'il est encore vivant!) et son fils Autéas auront droit à la récolte de céréales jusqu'au printemps 305, à celles de raisin, de fruits et d'olives jusqu'au printemps 304 (tableau I).

Tableau I.

\section{Calendrier du bail d'Aixonè}

\begin{tabular}{|c|}
\hline $\begin{array}{l}\qquad \text { juillet } 346-\text { juin } 345 \text { : archontat d'Archias } \\
\text { ANTHIAS locataire jusqu'en juin } 345 \text {, soit la fin de l'archontat d'Archias } \\
\text { mai-juin } 345 \text { : ANTHIAS fait sa dernière moisson }\end{array}$ \\
\hline $\begin{array}{l}\text { juillet } 345 \text { - juin } 344: \text { archontat d'Euboulos } \\
\text { juillet } 345 \text {, intallation des nouveaux locataires AUTOCLĖS ET AUTÉAS } \\
\text { été-automne } 345: \text { l'ancien locataire ANTHIAS récolte ses fruits et vendange } \\
\text { octobre-novembre } 345: \text { l'ancien locataire ANTHIAS récolte ses olives } \\
\text { novembre } 345: \text { coupe des oliviers, puis AUTOCLĖS ET AUTÉAS labourent et sèment } \\
\text { mai-juin } 344 \text { : AUTOCLĖS ET AUTEAS font leur première moisson }\end{array}$ \\
\hline $\begin{array}{c}\text { juillet } 344 \text { - juin } 343: \text { successeur d'Euboulos } \\
\text { été-automne } 344: \text { AUTOCLES ET AUTÉAS font leur première récolte de vigne et de fruits }\end{array}$ \\
\hline
\end{tabular}

On voit donc que le calendrier du bail, qui est le calendrier civil, prend néanmoins en compte les rythmes du calendrier agricole, en laissant à l'ancien locataire la possibilité de récolter les principaux fruits de la dernière année de son bail.

D. R.

19. Cf. AmouretTi, 1986, p. 5 I et 58 pour le sens précis d'arotos. 


\section{BIBLIOGRAPHIE}

AmouretTI, Marie-Claire, Le pain et l'buile dans la Grèce antique, Paris, Les Belles-Lettres, "Annales Littéraires de l'Université de Besançon 328", I986, p. 52 et 59;

-, "Oléiculture et viticulture dans la Grèce antique", in WELLS, Berit, (éd.), Agriculture in Ancient Greece, Stockholm, Paul Aströms Förlag, 1992, p. 77-86.

Behrend, Dietrich, Attische Pachturkunden, Munich, I970, notamment p. 80-82.

Bertrand, Joëlle, et Brunet, Michèle, Les Athéniens, Paris, A. Colin, 1993, p. 174-178.

Eliot, C. W. J., The Coastal Demes of Attica, 1962, p. Io-16 (origine de l'inscription).

WHITEHEAD, David, The Demes of Attica, 1986, notamment p. 152-159 (sur les baux des dèmes).

54 .

177 avant J.-C.

Contrats de location concernant quelques domaines appartenant à Apollon, Délos (Cyclades)

Éditions du texte : DURRBACH, Félix, Inscriptions de Délos, Paris, Librairie Champion, 1929, $\mathrm{n}^{\circ} 452$ et $\mathrm{n}^{\circ}$ 467. Raccord entre les deux fragments, lignes 16 à $25:$ BRUNET, Michèle, Bulletin de correspondance hellénique, II4, I990, p. 678-679. Restitution des lignes 26-29 et date de l'inscription : REgER, Gary, Hesperia, 63, 1994, p. IO5-IIo (nous modifions ici légèrement sa restitution de la l. 26, d'après Philippe GauTHIER, REG, I08, 1995, p. 498, nº 438).

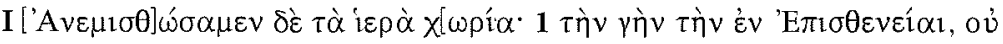
$\left.\kappa \alpha \theta 1 \sigma \tau \alpha \dot{v} v \tau \varsigma_{1}\right]$

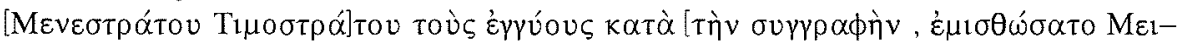

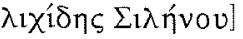

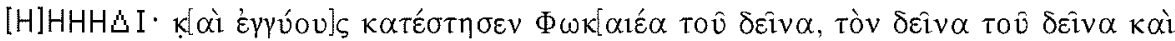
$\pi \alpha-]$

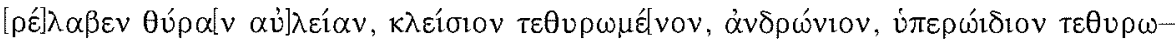

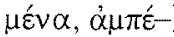

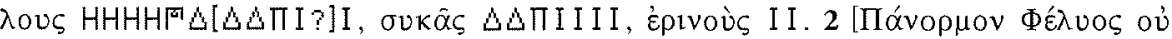

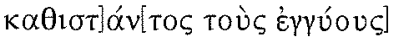

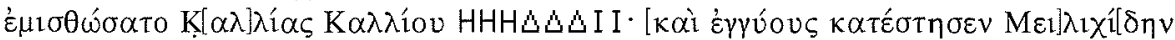

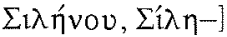

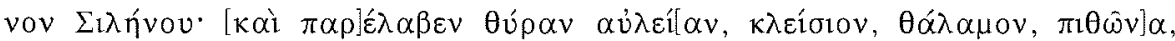

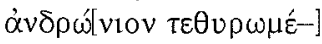

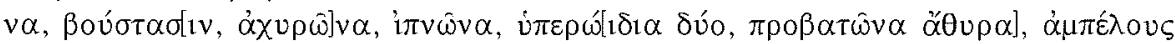
[XHHP $\triangle \Delta \Delta \Delta \Pi$ I I I],

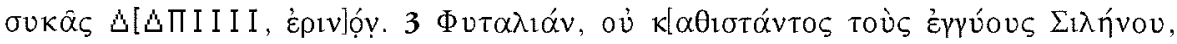

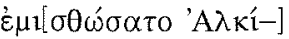

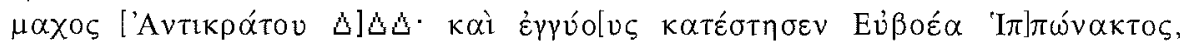
'A $\mu[$. .Tôे $\delta \varepsilon \hat{\imath} v \alpha \ldots . . . . . . .$.$] .$

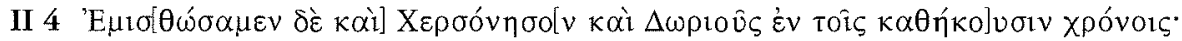
$\kappa \alpha\lfloor\varepsilon \dot{\varepsilon} \mu \theta \sigma \omega \sigma \alpha \tau o \mathrm{M} \varepsilon \lambda \eta \dot{\sigma} \sigma \tau-]$

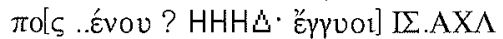

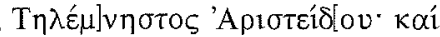

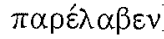




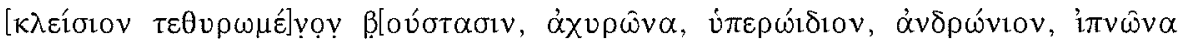

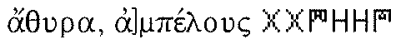

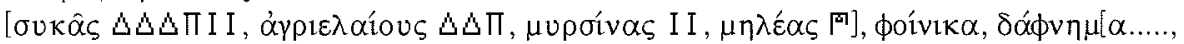

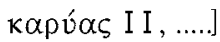

\section{Traduction}

I. Nous avons remis en location les domaines sactés;

1. la terre à Épisthéneia ${ }^{1}$ : étant donné que Ménestratos, fils de Timostratos, n'a pas présenté ses garants conformément à la réglementation, Meilichidès, fils de Silènos, l'a louée pour 4II drachmes. Il a présenté comme garants Phocaieus, fils de [...] et [...] fils de [...]. Il a reçu une porte de cour, une grande salle munie d'une porte, un local pour travailleurs masculins et un premier étage munis d'une porte, 487 (ou 488 ou 489) pieds de vigne, 29 figuiers et 2 figuiers sauvages.

2. Panormos ${ }^{2}$ : étant donné que Phélys n'a pas présenté ses garants, Callias, fils de Callias, l'a loué pour 332 drachmes. Il a présenté comme garants Meilichidès, fils de Silènos, et Silènos, fils de Silènos. Il a reçu une porte de cour; une grande salle, une chambre, un cellier $^{3}$, un local pour travailleurs masculins munis d'une porte; une étable, une grange pour la paille, un fournil ${ }^{4}$, deux premiers étages, une bergerie, tous sans porte; I 298 pieds de vigne, 29 figuiers, I figuier sauvage.

3. Phytalia ${ }^{5}$ : étant donné que Silènos n'a pas présenté ses garants, Alcimachos, fils d'Anticratès, l'a loué pour 30 drachmes. Il a présenté comme garants Eubocus, fils d'Hippônax, et Am[...], fils de [...].

II. 4. Nous avons mis en location Chersonèsos et Dorion ${ }^{6}$, à échéance normale. Mélèsippos, fils de [...]enos, l'a loué pour 3ro drachmes. Garants [...] et Tèlemnestos, fils d'Aristeidès. Il a reçu une grande salle munie d'une porte; une étable, une grange pour la paille, un premier étage, un local pour travailleurs masculins, un fournil, tous sans porte; 2750 pieds de vigne, 37 figuiers, 25 oliviers sauvages, 2 myrtes, 50 pommiers, un palmier, [?] laurier(s), 2 amandiers.

\section{Commentaire}

Ce texte n'est pas, comme les deux précédents, un document isolé mais un "extrait" d'une série provenant de Délos, îlot au cœur des Cyclades qui passait pour être le lieu de naissance d'Apollon. Sur cette île minuscule où se trouvait son sanctuaire et sur les deux îles voisines de Rhénée et Myconos, le dieu possédait une vingtaine de domaines agricoles qui étaient administrés par des magistrats chargés de la fortune sacrée?. Ces der-

1. Domaine sis à Délos.

2. Domaine sis à Rhénée.

3. Littéralement, "le local où sont les pithoi». Ces grosses jarres généralement enterrées - qui ressortissent pour cette raison à l'immobilier - contenaient des denrées liquides ou solides.

4. Littéralement, "le local où se trouve le four".

5. Domaine sis à Délos.

6. Domaine unique sis à Mykonos, sans doute formé par la réunion de deux anciennes propriétés privées.

7. Avant 3I4, ce sont des magistrats athéniens appelés amphictyons; puis, durant l'Indépendance, 
niers étaient tenus à une reddition de comptes annuelle et l'usage voulait que l'on gravât le bilan détaillé de leur exercice financier sur des stèles de marbre qui étaient exposées dans le sanctuaire. C'est ainsi que nous est parvenu un dossier exceptionnel concernant la gestion des propriétés agricoles de l'Apollon délien, qui comprend environ 70 documents inégalement échelonnés entre la fin du ve et le milieu du Ire siècle avant J.-C. ; ces textes se répartissent en trois catégories bien distinctes :

- deux documents exposent sous la forme de contrats-types l'ensemble des conditions réglementant tous les aspects de la prise en location. Le premier, communément désigné sous le nom de "hiéra syngraphè »8 ("contrat sacré»), est daté de 300 avant J.-C. et fut donc édicté peu après l'Indépendance de la cité de Délos acquise en 3I49; le second10, daté de $157 / 6$, modifie certaines des dispositions antérieures, à la suite de la perte d'autonomie de la cité délienne devenue possession athénienne en I66 avant J.-C.;

- la plupart des documents sont des listes récapitulant les recettes de fermages versées par les locataires du dieu;

- enfin, quelques stèles consignent sous une forme abrégée les contrats de location qui étaient conclus après mise aux enchères publiques, selon une périodicité normalement décennale durant l'Indépendance, mais quelquefois à des intervalles moindres en cas de défaillance du locataire en titre.

C'est donc à ce dernier groupe que se rattachent les quatre exemples que nous présentons. Un commentaire exhaustif de ces lignes nécessiterait de nombreux feuillets; c'est pourquoi nous limiterons ici nos remarques aux points essentiels, en privilégiant les aspects sociologiques et économiques, dans la mesure où les inscriptions déliennes font partie des très rares documents grecs qui autorisent de telles analyses.

\section{Aspects juridiques}

En se référant explicitement à la réglementation en vigueur (1. 17), les hiéropes ont procédé à la réadjudication (anamisthôsis) de trois domaines au terme de la troisième année de bail (I, $\$ I-3), et à l'adjudication (misthôsis) d'un domaine situé à Myconos (II, $\$ 4$ ). Afin de bien différencier les deux procédures, ils précisent que la mise en location du domaine myconiate s'est effectuée "à échéance normale» (1. 26), par opposition aux trois adjudications précédentes qui sont intervenues avant l'issue normale des baux. De fait, les domaines de Mykonos, «acquis » plus récemment par le dieu, étaient affermés les années de millésime 7 , alors que les contrats pour Délos et Rhénée étaient conclus les années de millésime o.

C'est la même cause qui a conduit à l'éviction des trois fermiers (contrats I à 3) : ils n'ont pas été en mesure cette année-là de fournir de nouvelles cautions, alors que la hiéra

ces magistrats se nomment hiéropes. Outre les biens-fonds ruraux, ils gèrent des immeubles en ville, afferment des taxes sur un certain nombre de services et perçoivent des intérêts sur les prêts; enfin, ils tiennent l'inventaire de l'ensemble du mobilier déposé dans les bâtiments du sanctuaire.

8. Inscriptions de Délos, 1929, 503. Ce contrat-type nous est malheureusement parvenu fort mutilé; dans les parties qui en sont conservées sont essentiellement envisagées les obligations financières du preneur.

9. Auparavant, la cité et le sanctuaire étaient de fait sous tutelle athénienne.

10. Inscriptions de Délos, 1929, I 4I6, B, I, I-56.

Histoire et Sociétés Rurales, $\mathrm{n}^{\circ}$ 9, $\mathrm{I}^{\mathrm{er}}$ semestre 1998, p. 2II-245. 
syngraphè obligeait à un renouvellement des garants11 - qui étaient ordinairement au nombre de deux - chaque année de bail. Une telle exigence du bailleur est, en l'état de nos connaissances, sans parallèle. Elle peut néanmoins s'expliquer en regard de la situation foncière un peu exceptionnelle qui existait à Délos : l'île tout entière a une superficie de 360 ha et, bien que ce finage minuscule fût complété par un territoire annexé plus grand, à Rhénée, on conçoit que la propriété de la terre ait été, de fait, dans la cité, un véritable privilège, réservé à un très petit nombre de citoyens ${ }^{12}$. Dans un tel contexte, la vingtaine de domaines offerts à la location par le dieu - qui était sans conteste le plus gros propriétaire de la cité - constituait une possibilité d'accès à la terre pour tout un groupe de citoyens qui n'étaient pas propriétaires, mais qui disposaient des moyens pour investir dans l'activité agricole, socialement valorisante et économiquement rentable. Indice de l'intérêt que ces Déliens portaient aux domaines "sacrés ", on a pu démontrer de façon convaincantel3 qu'une compétition entre candidats à la location avait été à I'origine d'une flambée artificielle des fermages à l'extrême fin du IVe siècle avant J.-C., qui entraîna une série d'impayés sanctionnés par des saisies. C'est donc vraisemblablement pour se prémunir contre de tels risques que la législation délienne édictée peu après était bien plus sévère qu'ailleurs, notamment à propos des cautions. En effet, les enjeux économiques à l'arrière-plan de la location des propriétés sacrées étaient à Délos bien plus considérables que dans d'autres cités : la rente foncière alimentait pour moitié le trésor d'Apollon, qui devait financer de lourdes dépenses pour le culte et l'entretien d'un grand sanctuaire de renommée internationale, mais qui fonctionnait aussi comme une banque de prêt, à laquelle la cité comme les particuliers avaient souvent recours.

\section{Sociologie des fermiers et de leurs garants}

Grâce à l'étude fondée sur une analyse prosopographique approfondie que Claude Vial a consacrée à la société délienne de l'époque de l'Indépendance ${ }^{14}$, il est possible de situer la plupart des fermiers d'Apollon dans leur lignée familiale et de connaître leurs activités publiques. Il apparaît ainsi qu'il y avait à Délos des «familles de fermiers " qui, contournant la réglementation conçue pour assurer une rotation régulière des preneurs ${ }^{15}$, investirent dans la constitution d'un cheptel et d'une main-d'œuvre agricole ser-

11. Leur solvabilité personnelle faisait l'objet d'un contrôle, car ils étaient responsables sur leurs biens propres.

12. On a calculé que les citoyens étaient entre I 200 et I 500 au début du IIe siècle avant J.-C., pour une population totale d'environ 6000 personnes, alors qu'on peut estimer par un calcul théorique que le territoire délien (sans Rhénée) ne pouvait comprendre qu'une cinquantaine d'exploitations de taille "normale» (entre 4 et 5 ha).

13. Voir REGER, I994, P. 2I5.

14. VIAL, I984.

15. La durée décennale des contrats semble avoir été en vigueur dès les débuts de la mise en location des domaines sacrés au ve siècle, à une époque où des étrangers (surtout Athéniens), tout autant que des Déliens, pouvaient en bénéficier; elle fut maintenue dans la législation délienne de 300 , vraisemblablement dans le but théorique d'éviter un accaparement de la propriété agricole sacrée sur une trop longue période par un petit nombre de personnes. La rotation régulière des preneurs était en outre garantie par le principe même de l'adjudication, mais surtout par la règle de l'épidékaton, qui autorisait la reconduction du bail moyennant une augmentation de $10 \% \mathrm{du}$ fermage antérieur : un tel pourcentage, qui diminuait considérablement la marge de profit du preneur en quelques décennies, ne pouvait que limiter rapidement ses velléités de se maintenir dans les lieux. 
vile16, et gardèrent un même domaine en location durant plusieurs générations, tout en s'arrangeant pour louer en même temps des exploitations proches ${ }^{17}$. L'activité agricole associée à l'élevage procurait en effet des revenus substantiels dans une île où, faute de disposer d'un finage étendu, une part de la population plus importante que dans d'autres cités ne pouvait prétendre vivre de l'autoconsommation. Ainsi, parmi les fermiers suffisamment riches pour avoir exercé des liturgies, près de la moitié appartiennent à quatre de ces familles dont les revenus provenaient essentiellement de la location de domaines sacrés. Conformément à la hiérarchie sociale qui, dans les cités de la Grèce antique, réservait les plus hautes fonctions administratives aux propriétaires fonciers, les 250 fermiers connus entre le IVe et le milieu du II ${ }^{e}$ siècle n'ont pas eu une aussi belle carrière des honneurs que les propriétaires fonciers : seulement un peu plus de rr\% d'entre eux ont occupé une magistrature durant le temps où ils étaient fermiers du dieu, contre $50 \%$ des propriétaires.

L'examen détaillé des quelques exemples présentés ici confirme pleinement ce constat. Il est néanmoins intéressant d'étendre l'enquête à la personnalité sociale des garants, car les liens qui les unissent aux fermiers éclairent les stratégies d'alliances familiales, les solidarités économiques entre citoyens partageant un même niveau de fortune, et le rôle fondamental que jouait la prise en location des domaines sacrés dans le fonctionnement économique, social et politique de la petite cité délienne (tableau 2).

\section{Composition et valeur locative des domaines agricoles}

Les études consacrées aux états des lieux qui accompagnent les contrats de location abrégés nous dispensent de revenir sur leur analyse ${ }^{18}$. Ces énumérations de locaux n'autorisent aucune reconstitution frable de la configuration d'ensemble des fermes ni des dispositions relatives des pièces qui les constituent. Car l'ordre des mentions obéit le plus souvent non pas à une logique descriptive, mais à un principe de rédaction qui tend à regrouper les locaux selon qu'ils sont ou non pourvus d'une porte au moment de l'entrée en jouissance : cet équipement était en effet considéré comme du mobilier par les Grecs, et sa fourniture par le bailleur était donc facultative.

Ces inventaires, destinés à permettre un contrôle du maintien en l'état de la propriété au moment de la reprise, montrent que l'on ne pratiquait pas systématiquement l'élevage du petit bétail sur tous les domaines d'Apollon ${ }^{19}$ où, par contre, la polyculture était de règle 20 : vignes et vergers y voisinaient avec des champs de céréales, mais, comme à Amorgos dans le domaine de Zeus Téménitès ${ }^{21}$, on constate l'absence des oliviers sur les exploitations sacrées, à Délos comme à Rhénée22. Sur Délos même, le vignoble était peu

16. Dans la pratique, les domaines étaient confiés à des régisseurs qui résidaient sur l'exploitation et dirigeaient les travaux effectués par des esclaves.

17. VIAL, I984, p. 317-338.

18. Voir KENT, I948, et, en dernier lieu, BRUNET, 1990, ainsi que, dans le présent volume, les réflexions de Christophe CHANDEZON à propos des bâtiments mentionnés dans les contrats de Mylasa.

19. Ici, seul le domaine Panormos à Rhénée possède une bergerie (probatôn), 1. 23.

20. Phytalia fait de ce point de vue exception.

21. Cf. document 52 et le commentaire afférent.

22. Seuls les domaines de Myconos comprennent des oliviers, mais on notera qu'il s'agit d'oliviers non greffés (1. 29), dont les fruits fournissaient une huile souvent utilisée comme excipient 


\section{Tableau 2. \\ Sociologie des locataires et de leurs garants}

\section{MEILICHIDÉs, fils de Silènos et SilÈNOS, fils de Silènos \\ sans doute secrétaire de la cité vers 170 biérope en 179}

Il s'agit de deux frères. Meilichidès devient fermier d'Épisthéneia (contrat I), au moment où son frère Silènos est déchu de la location de Phytalia (contrat 3). Tous deux se portent caution de Callias, preneur de Panormos (contrat 2). L'incapacité de Silènos à présenter de nouveaux garants ne doit certainement pas être interprétée comme le signe de sa ruine, car sinon, il n'aurait pas été accepté lui-même comme garant d'un tiers. La solidarité entre les deux frères s'exprime dans leur association comme garants, mais peut-être aussi dans l'abandon (volontaire?) d'un domaine peu rentable par l'un, alors que l'autre prend en location l'un des plus gros domaines de Délos.

\section{ALCimaCHOS II, fils d'Anticratès IV}

Le repreneur de Phytalia (contrat3) appartient à une famille de hauts magistrats qui ont presque tous été fermiers d'Apollon depuis le III siècle (cf. VIAL, I984, stemma familial XX, p. 2I8-219).

\section{Euboeus, fils d'Hipponax}

Garant d'Alcimachos pour la location de Phytalia (contrat 3), ce propriétaire de terres agricoles fut biérope en I8I. Il emprunta 500 drachmes à la caisse sacrée en 179 , en hypothéquant des biensfonds, et son fils Amphiclès se porta alors garant de cet emprunt. Par conséquent, il serait tentant de voir un autre effet de cette solidarité entre père et fils en restituant le nom d'Amphiclès, fils d'Euboeus, comme second garant d'Alcimachos, à la fin de la l. 25 (la restitution convient pour le nombre de lettres manquantes après le début conservé $\mathrm{AM}$ ).

\section{TĖLEMNESTOS II, fils d'Aristeidès II}

Il s'agit ici d'une caution prestigieuse apportée au locataire d'un domaine de Myconos (contrat 4). Ce personnage fut en effet l'orateur le plus en vue de la cité dans le premier tiers du IIe siècle ; il domina toute la vie politique de l'île, en étant le conseiller exclusif de l'Assemblée pour les questions de politique internationale, et créa ainsi de fait "un régime de démocratie dirigée" (Claude VIAL, 1984, p. 279). Sans avoir jamais été lui-même fermier du dieu, il ne dédaignait donc pas s'impliquer dans la vie économique en offrant sa garantie financière.

développé et l'arboriculture était, semble-t-il, beaucoup plus diversifiée à Myconos que sur les deux autres îles.

Pour expliquer les différences de redevances entre les domaines et leurs fluctuations hétérogènes dans le temps, on a depuis longtemps cherché à établir un rapport entre la valeur $\mathrm{du}$ fonds, dont témoignent partiellement ${ }^{23}$ ces états des lieux, et les montants des fermages. On évaluera la difficulté de cette entreprise en consultant le petit tableau établi à partir de l'échantillon traduit (tableau 3).

Si l'on excepte Phytalia qui est un «domaine» un peu particulier, vraisemblablement un jardin situé dans la ville ou sur ses franges immédiates, on constate que, curieusement, les montants des fermages sont inversement proportionnels au nombre de pieds de vigne que possèdent les exploitations. Outre les facteurs purement économiques qui

pour la fabrication du parfum. À propos de l'oléiculture à Délos, voir le bilan dressé par BRUN et BRUNET, 1997 .

23. Ils passent en effet sous silence les terres arables. 
Tableau 3.

Comparaison des redevances et des plantations en 177 avant J.-C.

\begin{tabular}{|c|c|c|c|}
\hline Nom du domaine & Localisation & Fermage en drachmes & Plantations \\
\hline Episthéneia & Délos & $4 \mathrm{II}$ & $\begin{array}{c} \pm 487 \text { pieds de vigne } \\
29 \text { figuiers }\end{array}$ \\
\hline Panormos & Rhénée & 332 & $\begin{array}{l}\text { I } 298 \text { pieds de vigne } \\
29 \text { figuiers }\end{array}$ \\
\hline Chersonèsos et Dorion & Myconos & 310 & $\begin{array}{c}2750 \text { pieds de vigne } \\
37 \text { figuiers } \\
25 \text { oliviers sauvages } \\
50 \text { pommiers } \\
\text { et quelques autres arbres }\end{array}$ \\
\hline Phytalia & Délos & 30 & Aucune \\
\hline
\end{tabular}

ont été invoqués pour expliquer ce phénomène paradoxal24, on n'a peut-être pas suffisamment pris en compte les différences de localisation des propriétés : quoique tout relatif, l'éloignement des terres rhéniotes et myconiates pourrait bien avoir aussi joué un certain rôle, qui expliquerait qu'une plus-value ait été attribuée par contraste aux domaines situés à Délos même, en raison de leur plus grande proximité par rapport au lieu de résidence des preneurs et à l'agora, où se vendaient les denrées agricoles.

En tout état de cause, cet exemple illustre les limites que rencontre toute analyse des phénomènes économiques dans l'Antiquité grecque : quand bien même nous disposons de données chiffrées comme dans ce cas précis, nombre de paramètres essentiels - qui ne relèvent certainement pas tous de la cliométrie - continuent à nous échapper.

M. B.

\section{BIBLIOGRAPHIE}

BRUN, Jean-Pierre, et BrunET, Michèle, "Une huilerie du premier siècle avant J.-C. dans le Quartier du théâtre à Délos ", Bulletin de correspondance helléniqque, 121, 1997, p. 573-616.

BRUNET, Michèle, "Contribution à l'histoire rurale de Délos aux époques classique et hellénistique ", Bulletin de correspondance hellénique, II4, I990, p. 669-682.

Étienne, Roland, et SAblé, Véronique, "Compte rendu de Gary Reger, 1994», Topoi, 5/2, I995, p. 555-560.

Kent, John H., "The Temple Estates of Delos, Rheneia and Myconos", Hesperia, 17, 1948, p. $243-338$.

REGER, Gary, Regionalism and Change in the Economy of Independant Delos, 3I4-I67 B.C., Berkeley, University of California Press, 1994, 396 p., 12 fig., 36 tableaux.

VIAL, Claude, Délos indépendante (3I4-I67 avant J.-C.). Étude d'une communauté civique et de ses institutions, Athènes, École française d'Athènes, Bulletin de correspondance hellénique, Supplérnent, $\mathrm{X}, \mathrm{1984}, 423 \mathrm{p}$.

24. On trouvera une discussion approfondie de cette question dans REGER, 1994, p. 189-247, à nuancer d'après ÉTIENNE et SABLÉ, I995. 


\section{5 . \\ IVe siècle avant J.-C. \\ Borne-témoin d'une hypothèque prise sur un domaine, Naxos (Cyclades)}

Principales éditions : Inscriptiones graecae, XII, Suppl. 194; FINLEY, Moses I., Studies in Land and Credit in Ancient Athens, soo-20o BC. The Horos-Inscriptions, New Brunswick, New Jersey, Rutgers, 1951, $\mathrm{n}^{\circ}$ 131; Nouveau choix d'inscriptions grecques, Lyon, Institut Fernand-Courby, 1971, $\mathrm{n}^{\circ} 25 \mathrm{D}$ (avec un commentaire en partie erroné).

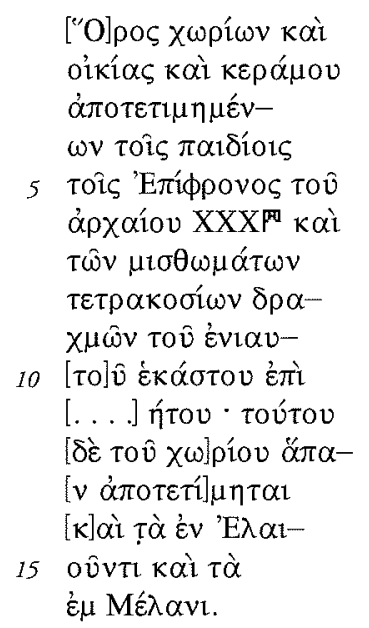

\section{Traduction}

Borne des terrains, de la maison et de la toiture en tuiles, hypothéqués au profit des enfants d'Épiphron, pour un capital de 3500 drachmes et pour les loyers d'un montant annuel de 400 drachmes, sous l'archontat de [...]êtos. La totalité de cette propriété a été donnée en hypothèque, ainsi que les terrains situés à Élaionte et à Mélas.

\section{Commentaire}

Cette petite stèle de marbre inscrite, haute d'environ $30 \mathrm{~cm}$, servait de borne (horos) signalant que le terrain sur lequel elle se trouvait était hypothéqué, et constituait une partie de la caution donnée pour pouvoir prendre en location des biens-fonds appartenant à des mineurs orphelins.

Ce document est un témoignage sur l'affermage de terres agricoles entre personnes privées, mais il concerne un cas très spécifique. La location des terres appartenant aux orphelins avait un caractère officiel, puisqu'elle était placée sous l'égide d'une des plus hautes autorités de la cité, comme le montre un passage de la Constitution des Athéniens d'Aristote : «L'archonte est chargé d'affermer les biens des mineurs et des épiclères ${ }^{1}$ jusqu'à leur quatorzième année, et il prend les hypothèques sur les biens des fermiers»

1. Fille orpheline de père et dépourvue de frère, donc héritière d'un patrimoine. 
(LVI, 7). La totalité des biens dont héritaient les orphelins pouvait être louée par adjudication (misthôsis oikou), soit par suite d'une décision testamentaire du défunt, soit par décision de leur tuteur ou à la demande d'un tiers. En garantie du paiement des fermages et du maintien de la valeur du capital foncier - qui devait être restitué à la majorité des enfants -, le preneur était tenu de fournir une hypothèque sur ses biens propres, comme dans les procédures d'emprunt. Cette possibilité de location intéressante - car elle ne nécessitait aucun investissement de la part du preneur, qui disposait de la main-d'œuvre et éventuellement du cheptel attachés au domaine - était dans la pratique réservée à des citoyens riches, nécessairement eux-mêmes propriétaires fonciers. Ici, le locataire des biens des enfants d'Épiphron a dû hypothéquer en leur faveur non seulement la totalité de la propriété marquée par cette borne, mais aussi des terrains agricoles situés en deux autres points de l'île de Naxos.

M. B. et D. R. 
